\title{
Explicit wave action conservation for water waves on vertically sheared flows
}

\author{
B.E. Quinn ${ }^{\mathrm{a}}$, Y. Toledo ${ }^{\mathrm{a}}$, V.I. Shrira ${ }^{\mathrm{b}}$ \\ ${ }^{a}$ School of Mechanical Engineering, Tel Aviv University, Israel \\ ${ }^{b}$ School of Computing and Mathematics, Keele University, UK
}

\begin{abstract}
This paper addresses a major shortcoming of the current generation of wave models, namely their inability to describe wave propagation upon ambient currents with vertical shear. The wave action conservation equation (WAE) for linear waves propagating in horizontally inhomogeneous vertically-sheared currents is derived following Voronovich (1976). The resulting WAE specifies conservation of a certain depth-averaged quantity, the wave action, a product of the wave amplitude squared, eigenfunctions and functions of the eigenvalues of the boundary value problem for water waves upon a vertically sheared current. The formulation of the WAE is made explicit using known asymptotic solutions of the boundary value problem which exploit the smallness of the current magnitude compared to the wave phase velocity and/or its vertical shear and curvature; the adopted approximations are shown to be sufficient for most of the conceivable applications. In the limit of vanishing current shear, the new formulation reduces to that of Bretherton \& Garrett (1968) without shear and the invariant is calculated with the current magnitude taken at the free surface. It is shown that in realistic oceanic conditions, the neglect of the vertical structure of the currents in wave modelling which is currently universal might lead to significant errors in wave amplitude. The new WAE which takes into account the vertical shear can be better coupled to modern circulation models which resolve the three-dimensional structure of the uppermost layer of the ocean.
\end{abstract}

Keywords: Wave Action Equation, Vertically Sheared Currents, Wave Rays

and their coupling with currents is also a way towards improvement of the weather prediction and climate models [2].

The primary goal of this work is to put forward an "explicit" closed form of the wave action conservation equation (WAE) suitable for operational forecasting which takes into account the vertical shear of the ambient currents. The seminal work of Bretherton and Garrett [3] examined linear wavetrains in a moving media and deduced that it is the adiabatic invariant, which they called wave action (not the wave energy) that is conserved. They applied their fundamental idea of the wave action conservation to a large variety of waves, such as, e.g., sound waves, Alfvèn waves, internal gravity waves and inertial waves, Rossby waves, etc [3]. For the problem focused upon here, i.e. surface gravity waves propagating on currents, the co-existence of motions of vastly different scales in natural water basins presents a serious challenge for their direct numerical modeling. On the other hand, for water waves on currents in nature, the almost universal vast separation of spatial and temporal scales provides a possibility for developing an asymptotic description of the coupled evolution of waves and currents. To the leading order, the wave dynamics is captured by fast and short linear waves, while the evolution of the currents is devoid of fast and short scales. In the present work we focus entirely on the dynamics of such wave fields. Thus, we 
are interested in the phase averaged evolution of linear wave fields on the time and space scales shorter than those where nonlinear interactions become important. This range of scales is quite substantial. For example, for dominant wind waves this range is typically up to tens of minutes and kilometers, for swell it is up to tens of kilometers. At these scales inhomogeneities due to currents and topography are the dominant factors.

Here we assume an arbitrary current profile with non-uniform vorticity and exploit the scale separation. To this end, the WKBJ approach is employed following the mostly overlooked work by Voronovich [4] (hereafter V76), where the WAE for linear surface and internal gravity waves on shear flows in a fluid of arbitrary depth was first derived. The significance of this work goes beyond the mere derivation of the WAE for the generic situation; it also demonstrated the role of the equations for lowfrequency larger scale motions and, hence, the factors which might have negligible direct effect on waves, but are of importance for the mean flows and through this back door, on wave action conservation. Independently, White [5] arrived at a similar derivation of the WAE but confined to deep water waves only. Both derivations yield an equation governing slow evolution of wave amplitude in space and time in an implicit form. To use it the eigenvalues (frequencies) and eigenfunctions (vertical modes) have to be found in each point of a wavepacket trajectory in "slow" space, which requires solving the boundary value problem for waves on a vertically-sheared current (the Rayleigh equation and the appropriate boundary conditions on the free surface and bottom). Then the solutions of the boundary value problem have to be used to find the packet trajectory and substituted into the WAE. Exact analytical solutions of the boundary value problem for an arbitrary current are not known. Probably it is this impediment which prevented the adoption of Voronovich's findings in practical wave modelling. Here Voronovich's derivation is revisited highlighting the junctions in the derivation where taking into account some extra effects such as the Earth's rotation, ambient flow turbulence, wind forcing, etc., might also be important and result in a different WAE. A priori one could not rule out a noticeable effect of the Earth's rotation despite the significant scale separation, since the numerical simulations of turbulent Reynolds' stresses in sheared flow beneath the free surface show a significant effect caused by the rotation under the comparable scale separation [6]. In the present work, Earth's rotation is taken into account, while ambient flow turbulence and wind forcing are neglected. Still, they were discussed in order to outline where and how these effects might enter the problem.

Taking into account the presence of the vertical shear of the currents also substantially affects the nonlinear dynamics of the waves propagating on the currents. In particular, the wave's vertical structure differs from that for potential waves [7, $8]$, the timescale of the Benjamin-Feir instability $\left(O\left(\left(\mu^{2} \omega\right)^{-1}\right), \mu\right.$ is wave steepness, $\omega$ is wave frequency) changes (e.g. [9]) and triad resonant interactions between pairs of surface harmonics and a vorticity wave, which are absent in vertically uniform flows, become possible [10] on the timescale of $O\left((\mu \omega)^{-1}\right)$. It should be noted that for short wind waves of typical wavelength $\sim 0.1 \mathrm{~m}$, the nonlinear interactions can happen quite quickly but in this study we focus on wavelengths in the range $10-100 \mathrm{~m}$ and on linear dynamics of water waves on horizontally and vertically varying currents; the nonlinear interactions, both triad and cubic only have to be considered when attempting to describe wave evolution on longer timescales.

Although for the boundary value problem for waves on a current with an arbitrary vertical profile exact analytical solutions have not been found. Fortunately, in typical oceanic conditions there are always natural small parameters which can be exploited to get asymptotic solutions for generic profiles. Stewart and Joy [11] derived an approximate dispersion relation for deep water waves on a depth-dependent current as the leading order term in an asymptotic expansion, the current magnitude normalized by the wave phase velocity being the small parameter. This advance was followed by a finite-depth extension of this approach by Skop [12]. The second order term in this expansion was by found Kirby and Chen (1989). An alternative solution of the deep water boundary value problem in terms of a converging series was derived by Shrira [13] by exploiting the presumed smallness of vorticity and more recent work includes analysis of the boundary value problem for a piecewise linear approximation [14]

This paper brings together both lines of inquiry: the implicit WAE formulation of Voronovich [4], hereafter V76 and asymptotic solutions of the boundary value problem for waves on a sheared current. The V76 formulation is exact within the framework of the linearised Euler equations and the WKBJ approximation. Here we choose an approximate solution to the boundary value problem, most appropriate in our context, which makes the WAE explicit and balances the accuracy and simplicity. Thus, for an arbitrary vertical profile of the current we put forward a formulation of the WAE suitable for operational forecasting with an explicit wave action invariant for waves on a slowly varying current and topography. The discrepancies between the predictions of the new WAE and that for the vertically averaged currents, on the one hand, and the "exact" V76, on the other, are examined. We show that for sample realistic situations the adopted approximation indeed works well. The situations, where the discrepancy with the results for vertically averaged currents is significant, are identified.

Without any pretence at drawing a comprehensive review it makes sense to outline other lines of enquiry 
on water waves on shear currents to provide the context for this study. Most of the efforts concentrated on theoretical studies. As far as the linear theory is concerned the reviews by Peregrine [15] and Peregrine \& Jonsson [16] are still relevant today. Just a few developments having relevance to the current study have to be specially noted. Although the scale separation underpinning the universally adopted WKBJ approach practically always holds in the ocean, the caustics do occur. In the vicinity of a caustic the field evolution does not result in a singularity predicted by the ray theory but needs to be described by a special model equation. In the absence of vertical shear in the vicinity of a turning point the model equation is the standard Airy equation. For waves on a vertically sheared current the problem is more complicated but it has been solved by McKee [17, 18]. An independent derivation of the WAE for unidirectional waves on a linearly sheared collinear current was carried out by Jonsson et al. [19]. Since for this case it is possible to introduce a potential for the wave motion and the boundary value problem is straightforward to solve, no further approximations are needed, which makes it a very attractive toy model. We are not aware of it being applied to the modelling of any real situation in the ocean. The popularity of considering the constant shear currents does not seem to wane even in the linear setting; a recent paper by Ellingson and Brevik [20] provides an update. The mild-slope equation, widely-used in nearshore and coastal regions, very recently was extended to include the effects of a linearly-sheared current [21].

However, it is in the area of theoretical studies of nonlinear waves where the possibility of introducing a potential for waves on linearly sheared currents was most heavily exploited: the number of papers is now counted in hundreds. Here we mention just a few key nonlinear effects and phenomena discovered. In the presence of the vertical shear the shape of steady nonlinear waves differs from that in the absence of shear, often quite significantly (e.g. [7, 22]). This change of shape is a generic manifestation of vertical shear (e.g. [8]), but is much more difficult to explore without constant vorticity assumption. The presence of vertical shear also makes possible the existence of solitary waves on deep water, as was shown analytically [23] and numerically [24]. Surprisingly, although the steady solutions for periodic nonlinear waves on a horizontally uniform current with constant shear have been known for more than thirty years, to our knowledge there has been no attempt to derive a nonlinear conservation law and to generalise the results of Peregrine \& Thomas [25] for horizontally nonuniform currents and varying depth by taking into account constant vorticity. A major advance concerned with constant vorticity waves has been reported in [26], where the method of conformal mapping was extended to solve numerically fully nonlinear Euler equations for two-dimensional inviscid free-surface flows with constant vorticity over ar- bitrary nonuniform bottom profile in an extremely efficient and accurate way.

For a multitude of reasons the modulational instability of weakly nonlinear waves propagating upon horizontally uniform shear flows was a constant focus of attention for more than forty years and resulted in a large corpus of works. Most of the studies were aimed at deriving the nonlinear Schrödinger (NLS) type equation for the envelope of a weakly nonlinear wave train, the first such study by Johnson [27] which moulded the paradigm for all subsequent studies: an ideal fluid, a priori given current which does not evolve, a weakly nonlinear wavetrain, a narrowband wavetrain, with the narrowness chosen to be balanced by the nonlinearity. Johnson [27] considered strictly one-dimensional wave propagation on an collinear current with an arbitrary shear profile. The cycle of influential works by Benney and co-authors (see e.g. [9] and references therein) extended the consideration to two spatial dimensions. It has been established that for an arbitrary profile of the current the evolution of a weakly nonlinear narrowband wavepacket varying in two horizontal dimensions is governed by the NLS-type equation coupled with elliptic equations for the induced "mean" flow. The main conclusions of Benney's group can be briefly summarized as follows: the shear does affect the modulational instability and for a strong shear the effect might be substantial; crucially, for a strong shear the transverse instability is much stronger than the longitudinal one. This conclusion was somehow forgotten; the most studied proved to be strictly longitudinal modulations for two-dimensional motions. Again the simplicity of the constant vorticity and piecewise constant vorticity models proved to be irresistible, see e.g. Baumstein [28] and Thomas et al. [29] and references therein.

Models with a piece-wise constant vorticity profiles give rise to qualitatively new phenomena totally absent in constant vorticity models and are somewhat hidden in the models with continuous smooth vorticity profiles. The jumps of vorticity support interfacial waves, referred to as the vorticity modes, this makes possible resonant triad interactions between the surface waves and the vorticity modes, moreover, among these triad interactions there are explosive ones, that is, the interactions resulting in a finite time blow-up $[30,31]$. With the notable exception of the last two references all the above works were concerned with deterministic evolution of narrow band weakly nonlinear wave trains. Returning to the oceanic waves which are always random, rarely sufficiently narrow band to be described in terms of isolated wavetrains and never (except for laboratory tanks) are strictly one-dimensional, oceanic waves are necessarily described in terms of their statistical characteristics, primarily spectra. The only attempt we are aware of to incorporate the account of vertical shear into description of wave kinetics was undertaken in [10]. Nonlinear triad interactions between the surface and 
vorticity modes are not entirely an artifact of piecewise constant vorticity models. Such interactions were shown to be always present for arbitrary smooth vorticity profile, the corresponding interaction coefficients were derived for typical oceanic situations exploiting the weakness of the current with respect to the surface wave phase velocities; to describe evolution of wave spectra subjected to both the triad and standard quartic interaction the corresponding kinetic equation has been derived [10]. This line of inquiry did not get a direct continuation. The present work is complementary to it: we take into account the horizontal inhomogeneity of the shear currents and bottom profile concentrating on the smaller time scales. The next step needed is to integrate the two approaches, which represents a conceptual challenge: the resonant interactions are described in the wavevector space, while the effects due to spatial inhomogeneity captured by the WAE are naturally described in the coordinate space.

All mentioned theoretical works are based on the idea that there exist either an a priori given vertical profile of a current or, as in V76, it is specified by the Euler equations for the low-frequency motions upon which either linear and nonlinear waves evolve. To what extent this split occurs in reality is not known. The field measurement show very low vertical shear in the layer adjacent to the surface, which is attributed to the wave breaking [32]. At the moment it is not clear at what time scale the current profile adjusts to changes of wave breaking intensity controlled primarily by wave steepness. If/when the blow-ups predicted by Voronovich et al [31] does occur, it would locally destroy the shear flow vorticity profile, is also not clear what effect this will have on wave propagation and how long it takes for the profile to recover. Breaking of dominant waves, although rare, certainly temporarily destroys the vertical structure of the flow in a way poorly understood so far [33]. Sufficiently accurate field observations of wave evolution on horizontally and vertically varying currents with simultaneous high resolution measurements of the currents are beyond the reach of the existing techniques. Although in the laboratory the measuring capabilities are much better, the laboratory tanks rarely allow for two-dimensional wave propagation desirable for the effects of we are interested in to be pronounced. Hence, at present neither the field observations nor even the best tank experiments (e.g. [34]) can resolve the fundamental open questions of wave-current interactions. Being fully aware of these open questions we adopt the following approach, we focus on linear phase averaged dynamics of water waves on horizontally and vertically varying currents presumed to be given; the nonlinear interactions, both triad and quartic are neglected.

The layout of the paper is as follows: In $\S 2$, the separate dynamical equations are derived for the mean flow and the wave field. Under the standard separation of scales WKBJ assumptions, the derivation of the Rayleigh equation together with the invariant and group velocity of the WAE is revisited in $\S 3$ taking into account the Earth rotation. The derivation for the invariant and group velocity, which takes into account vertical shear is detailed in $\S 4$. In $\S 5$ the results are analysed to assess the improvement to the usual WAE due to taking into account vertical shear for a family of realistic-type vertical velocity profiles. The dynamics of the wave action is determined to large extent by the ray trajectories of the wave packets. The effect of the vertical shear on the wave ray paths and, hence, wave amplitudes is examined in $\S 6$. We briefly discuss the results and implications in $\$ 7$.

\section{Governing equations}

Our starting point is the Euler equations of motions for an incompressible fluid of constant density $\rho_{0}$ on a rotating frame with $f$ as the varying Coriolis parameter and a free-surface under the action of gravity. In the Cartesian frame $x, y, z$ with zero of the vertical coordinate $z$ on the unperturbed water surface, the motions are characterized by the horizontal velocity field $\overline{\mathbf{u}}=(\bar{u}, \bar{v})$, the vertical velocity field $\bar{w}$ and the free surface elevation $\bar{\eta}(x, y, t)$.

$$
\begin{aligned}
\frac{D \overline{\mathbf{u}}}{D t}+f \hat{z} \times \overline{\mathbf{u}}+\frac{1}{\rho_{0}} \nabla p & =0 \\
\frac{D \bar{w}}{D t}+g+\frac{1}{\rho_{0}} \frac{\partial \bar{p}}{\partial z} & =0 \\
\nabla \cdot \overline{\mathbf{u}}+\frac{\partial \bar{w}}{\partial z} & =0
\end{aligned}
$$

where $D / D t=\partial / \partial t+\overline{\mathbf{u}} \cdot \nabla+\bar{w} \partial / \partial z, \nabla \cdot={ }_{381}$ $(\partial / \partial x, \partial / \partial y)$ is the horizontal derivatives vector, $f$ is the Coriolis parameter. The neglect of surface tension implies that we are interested in wavelengths longer than a few centimeters while the neglect of bottom friction assumes that for linear theory, the turbulence generated by the bottom friction remains local and is not transported into the main flow. As a first approximation to reality we consider inviscid fluid, which is a fair assumption since our main interest is in the wave motion at relatively short timescales. These basic equations are supplemented with the usual kinematic and dynamic boundary conditions at the surface and the dynamic boundary condition at the bottom which are respectively,

$$
\begin{aligned}
\frac{\partial \bar{\eta}}{\partial t}+\overline{\mathbf{u}} \cdot \nabla \bar{\eta}-\bar{w} & =0, & & z=\bar{\eta} \\
\bar{p} & =0, & & z=\bar{\eta} \\
\bar{w}+\overline{\mathbf{u}} \cdot \nabla h & =0 & & z=-h
\end{aligned}
$$

at the free surface $z=\bar{\eta}(x, y, t)$ and at the bottom $z=-h\left(\varepsilon_{1} x, \varepsilon_{1} y\right)$.

In nature, the problem under consideration has a number of small parameters. Slowness of the horizontal spatial variation of the main flow compared to the characteristic wave scale, is characterised by a small 
parameter $\varepsilon_{1}$, while the slowness of the ambient current temporal variability compared to the characteristic wave period is characterised by an independent parameter $\varepsilon_{2}$. Note that $\varepsilon_{2} \ll \varepsilon_{1}$. Variation in the vertical is characterised by an independent parameter $\delta_{0}$ and its scaling is left unspecified for now.

All flow variables can be split into two components: the fast oscillating part $(\sim)$ and the slow non-wave motion $\left({ }^{(0)}\right)$. Thus a generic field variable $\bar{\zeta}$ can be presented as the sum

$\bar{\zeta}=\zeta^{(0)}+\tilde{\zeta}$

By inserting ansatz (2.3) into both the governing equations (2.1) and boundary conditions in (2.2) and retaining terms to the lowest order, the dynamics of the ambient current can be considered as the average or the overall motion. Ordering for the mean flow part is

$\zeta^{(0)}=\zeta^{(0)}\left(\varepsilon_{1} x, \varepsilon_{1} y, \delta_{0} z, \varepsilon_{2} t\right)$

and the resulting mean flow equations and boundary conditions (are expanded in a Taylor series to attain the mean and oscillatory parts on the free surface) are equivalent to those in Eq. (6) of V76 except here the Coriolis term enters into the horizontal momentum equations.

The time scales of the oscillatory dynamics are much shorter than that of the mean flow even in the case of a long swell. A typical wave celerity for dominant wind waves and swell is of $O\left(10 \mathrm{~ms}^{-1}\right)$. The Coriolis frequency is small and we characterise this smallness by an independent small parameter $\varepsilon_{3}$. To see what might be affected by rotation, we, for the time being, assume $\varepsilon_{3} \sim \varepsilon_{1}$, then the linearized equations to first order take the form:

$\frac{d \tilde{\mathbf{u}}}{d t}+\varepsilon_{1} \tilde{u}_{i} \frac{\partial \mathbf{u}^{(0)}}{\partial x_{i}}+\delta_{0} \tilde{w} \frac{\partial \mathbf{u}^{(0)}}{\partial z}$

$$
\begin{aligned}
+\varepsilon_{3} f \hat{z} \times \tilde{\mathbf{u}}+\frac{1}{\rho_{0}} \nabla \tilde{p} & =0 \\
\frac{d \tilde{w}}{d t}+\varepsilon_{1} \delta_{0} \tilde{w} \frac{\partial w^{(0)}}{\partial z}+\frac{1}{\rho_{0}} \frac{\partial \tilde{p}}{\partial z} & =0 \\
\nabla \cdot \tilde{\mathbf{u}}+\frac{\partial \tilde{w}}{\partial z} & =0
\end{aligned}
$$

with corresponding boundary conditions

$$
\begin{aligned}
\frac{d \tilde{\eta}}{d t}+\tilde{\mathbf{u}} \cdot \nabla \eta^{(0)}-\tilde{w}-\frac{\partial w^{(0)}}{\partial z} \tilde{\eta}=0, & z=\eta^{(0)} \\
\tilde{p}=-\frac{\partial p^{(0)}}{\partial z} \tilde{\eta}, & z=\eta^{(0)} \\
\tilde{w}+\varepsilon_{1} \tilde{\mathbf{u}} \cdot \nabla h=0, & z=-h .
\end{aligned}
$$

The bottom boundary condition (2.6c) follows by assuming the viscosity to be negligible for the wave dynamics and so becomes the free-slip condition in which mild-slope changes of the bottom have been incorporated.

\section{Wave action equation (WAE) with account ${ }_{451}$ of vertical shear}

Exploiting the naturally occurring separation of scales - "short" and "fast" waves vs "slowly" varying environment- the WKBJ asymptotic approach is employed, e.g. [35]. Seeking solutions as an asymptotic series

$\tilde{\zeta}=\Re \mathrm{e}\left\{\left[\zeta^{(1)}+\varepsilon_{1} \zeta^{(2)}+\ldots\right] e^{\mathrm{i} S(x, y, t)}+\ldots\right\}$,

where

$\frac{\partial S}{\partial t}=-\Omega=f(\mathbf{k}, \mathbf{x}, t), \quad \nabla S=\mathbf{k}$

the local wavevector $\mathbf{k}=\left(k_{x}, k_{y}\right)^{T}, k=|\mathbf{k}|$ is related to the local wavelength $\lambda$, by the standard relation $\lambda=\frac{2 \pi}{k}, S$ is the wave phase and $\Omega$ is the local angular frequency.

Substituting Eq. (3.1) into Eqs. (2.5) and (2.6) and retaining lowest order terms yields (with superscript 1 omitted):

$$
\begin{aligned}
-\mathrm{i} \sigma \mathbf{u}+\delta_{0} w \frac{\partial \mathbf{u}^{(0)}}{\partial z}+\frac{\mathrm{ik}}{\rho_{0}} p & =0 \\
-\mathrm{i} \sigma w+\frac{1}{\rho_{0}} \frac{\partial p}{\partial z} & =0 \\
\mathrm{i} \mathbf{k} \cdot \mathbf{u}+\frac{\partial w}{\partial z} & =0
\end{aligned}
$$

where $\sigma$ is the Doppler-shifted, depth-dependent fre- ${ }_{471}$ quency, defined as

$\sigma(z)=\Omega-\mathbf{k} \cdot \mathbf{u}^{(0)}$.

The quantity $\mathcal{U}$ is introduced to characterise the projection of the velocity onto the wavevector direction and $C$ for the wave celerity, defined as,

$\mathcal{U}(k, z)=\frac{\mathbf{k} \cdot \mathbf{u}^{(0)}}{k}, \quad C=\frac{\Omega}{k}$.

Standard manipulations of Eqs. (3.3) yield the Rayleigh equation for the vertical component of velocity (see e.g. [36])

$\frac{\partial^{2} w}{\partial z^{2}}-\left(k^{2}-\frac{\delta_{0}^{2}}{C-\mathcal{U}} \frac{\partial^{2} \mathcal{U}}{\partial z^{2}}\right) w=0$.

From Eqs. (3.3) and (3.6) all other components of ${ }_{482}$ velocity, $\mathbf{u}$ and pressure $p$ can be easily expressed in ${ }^{483}$ terms of $w$ as in Eq. (10) of V76. The corresponding $\quad{ }^{484}$ boundary conditions are

$$
\begin{aligned}
-\mathrm{i} \sigma \eta-w=0, & z=\eta^{(0)} \\
p=\rho g \eta, & z=\eta^{(0)} \\
w=0, & z=-h
\end{aligned}
$$

which allow the boundary condition at the free sur- ${ }_{489}$ face, $z=\eta^{(0)}$ to be expressed in terms of $w$ as

$\frac{\partial w}{\partial z}+\left(\frac{\delta_{0}}{(C-\mathcal{U})} \frac{\partial \mathcal{U}}{\partial z}-\frac{g}{(C-\mathcal{U})^{2}}\right) w=0$.

453

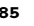

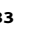

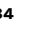

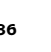

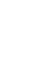

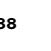

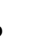


The local boundary value problem comprised of the Rayleigh Eq. (3.6) and the boundary condition (3.8) specifies the eigenvalues $C$ as functions of $\mathbf{k}$ and the local vertical mode structure $w(\mathbf{k}, z)$. The pressure and the horizontal velocities can then be expressed in terms of $w$ according to Eq. (10) of V76.

The phase of the wave $S$ by the Hamilton-Jacobi equation is

$$
\frac{\partial S}{\partial t}+f(\nabla S, \mathbf{x}, t)=0
$$

whose characteristics are the ray equations specifying positions of a wavepacket $\mathbf{x}$ and its wavevector $\mathbf{k}$
$\partial_{t} \mathbf{x}=\nabla \mathbf{k}$
$\partial_{t} \mathbf{k}=-\nabla \Omega$.

Hence, solutions of these equations prescribe ray trajectories, that is evolution in time of the position of a wavepacket and its central wavevector for given initial conditions, $\mathbf{x}(0), \mathbf{k}(0)$. We recall that at each point of the trajectory the vertical distributions of $w$ and local dispersion relation $\Omega(\mathbf{k})$ are given by the boundary value problem (Eqs. (3.6) and (3.8)).

To determine the evolution of wave amplitudes one must proceed to the next order. It is straightforward to derive similar equations for the next order (for details see Appendix B). To eliminate secular growth of the solutions of this second-order linear inhomogeneous boundary value problem it is necessary to impose a solvability condition, supplemented with the lowest-order mean flow equations, which yields the wave action conservation equation we are looking for

$$
\frac{\partial I_{v s}}{\partial t}+\nabla \cdot\left(\mathbf{C}_{\mathbf{g}} I_{v s}\right)=0
$$

where

$$
\begin{aligned}
I_{v s}= & -\int_{-h}^{\eta^{(0)}} \frac{1}{2 \sigma^{2} k^{2}} \frac{\partial^{2} \sigma}{\partial z^{2}} w^{2} d z \\
+ & {\left[\left(\frac{g}{\sigma^{3}}+\frac{1}{2 \sigma^{2} k^{2}} \frac{\partial \sigma}{\partial z}\right) w^{2}\right]_{z=\eta^{(0)}} } \\
\mathbf{C}_{\mathbf{g}} I_{v s}= & -\int_{-h}^{\eta^{(0)}}\left(\frac{1}{2 \sigma^{2} k^{2}} \frac{\partial^{2} \sigma}{\partial z^{2}} \mathbf{u}^{(0)}\right. \\
& \left.-\frac{1}{2 \sigma k^{2}} \frac{\partial^{2} \mathbf{u}^{(0)}}{\partial z^{2}}+\frac{\mathbf{k}}{k^{2}}\right) w^{2} d z \\
+ & {\left[\left(\left(\frac{g}{\sigma^{3}}+\frac{1}{2 \sigma^{2} k^{2}} \frac{\partial \sigma}{\partial z}\right) \mathbf{u}^{(0)}\right.\right.} \\
& \left.\left.-\frac{1}{2 \sigma k^{2}} \frac{\partial \mathbf{u}^{(0)}}{\partial z}+\frac{g \mathbf{k}}{\sigma^{2} k^{2}}\right) w^{2}\right]_{z=\eta^{(0)}}
\end{aligned}
$$

and the subscript "vs" is for vertical shear, denoting the exact invariant and group velocity. Dividing Eq. (3.13) by Eq. (3.11) gives an expression for the group velocity $C_{\text {gvs }}$. Note that under adopted scaling of the Earth's rotation $f$, i.e. the inverse Rossby number $\varepsilon_{3} \varepsilon_{1} \ll 1$, the taking into account of rotation in the Euler equations did not change the wave action equation of V76, although it has to be stressed that it enters the problem implicitly through equations for the mean flow Eqs. (2).

Thus, the problem of describing linear water waves on a slowly varying current with vertical shear has been reduced to finding the first-order variables $p, \mathbf{u}$, $C$ and $w$, the wave phase $S$ and the wave amplitudes from the WAE.

\section{Explicit formulation of the WAE}

In its current form, the WAE (3.10) is difficult to apply to operational wave models as it is too computationally intensive: it is required to solve the Rayleigh equation for every node, frequency, direction, etc. at every timestep. A simplification is required for it to be of practical use. To this end an asymptotic approximation to the WAE (3.10) will be detailed here and its accuracy subsequently compared with the exact adiabatic invariant and group velocity specified by Eqs. (3.11) and (3.13). This approximation takes into account vertically-varying currents and assumes a current to be weak compared to the wave phase velocity, which is a realistic assumption since dominant wind waves and swell have phase velocities which far exceed the speed of the current. The weak current approximation also implicitly assumes the smallness of the current gradient and curvature; in generic situations when the current profile is smooth, the smallness of the current magnitude also ensures sufficiently small gradient and curvature. It is convenient to characterize weakness of the current by a new nondimensional small parameter $\xi=\mathcal{U} / C$. The nondimensional parameter $\kappa=\mathcal{U}^{\prime} / \Omega$ denotes the smallness of gradient and $\epsilon_{5}=O\left(\mathcal{U}^{\prime \prime} / \Omega k\right)$ denotes the smallness of curvature. For generic smooth profiles all these small parameters are small and comparable and therefore could be denoted by $\varepsilon_{5}$. For the situations with very sharp gradients and large curvatures where $\mathcal{U}^{\prime \prime} / \Omega k \gg 1$ the expansion in $\xi$ is no longer valid, although as a rule it works well beyond the range of its asymptotic validity often even when $\mathcal{U}^{\prime \prime} / \Omega k \gg 1$. The reasons why are not clear and require a special consideration, which goes beyond the scope of this work. For a more detailed discussion of the nondimensional scaling, refer to [13].

An approximate dispersion relation for water waves on a generic depth-dependent current was first put forward by Stewart and Joy [11], which exploited presumed weakness of the current. Its extension to water of finite depth by Skop [12] was then continued to higher orders by Kirby and Chen [37]. Note that while these authors applied the multiscale expansion to the Rayleigh equation, which is just an element of the WAE, here it is applied to the WAE as a whole. An alternative solution to the deep water boundary value problem in terms of a converging series was derived by Shrira [13] by exploiting the presumed smallness of vorticity. In each case the choice of the best specific approximation is dictated by the context. 
Without loss of generality we assume a weak smooth current and solve approximately the Rayleigh boundary value problem Eqs. (3.6), (3.7b) and (3.7c) by expanding the wave celerity $C$, vertical velocity $w$ and the dispersion $\sigma$ in Eq. (D.12), the relation between the amplitude of the surface elevation $a$ and the amplitude of the vertical velocity $A$ as follows

$$
\begin{aligned}
\frac{\sigma}{k} & =C_{0}+\varepsilon_{5}\left(C_{1}-\mathcal{U}\right)+\varepsilon_{5}^{2} c_{2}+\ldots \\
w & =A\left(w_{0}+\varepsilon_{5} w_{1}+\varepsilon_{5}^{2} w_{2}+\ldots\right) \\
A^{2} & =k^{2}\left(C_{0}+\varepsilon_{5}\left(C_{1}-\mathcal{U}\right)+\ldots\right)^{2} a^{2}
\end{aligned}
$$

The expressions for $C_{0}$ and $C_{1}, w_{0}, w_{1}$ and $\sigma_{0}$ are equivalent to those found by Kirby and Chen [37] and are given in Appendix C. On applying these formulae to Eqs. (3.11)-(3.13), retaining terms to the lowest order and after some algebra, we find the approximated wave action and its flux,

$$
\begin{aligned}
I & =\left\{\frac{a^{2} g}{\sigma}\left(1+\varepsilon_{5} \mathcal{R}_{1}\right)\right\}_{z=\eta^{(0)}} \\
\mathbf{C}_{\mathbf{g}} \mathbf{I} & =\left\{\frac{a^{2} g}{\sigma}\left[C_{g \mathrm{nvs}} \frac{\mathbf{k}}{k}+\varepsilon_{5}\left(\mathbf{u}^{(0)}+\mathbf{R}_{2}\right)\right]\right\}_{z=\eta^{(0)}}
\end{aligned}
$$

where $C_{g_{\text {nvs }}}$ is the usual group velocity under the noshear approximation specified in Eq. (D.10), while the $O\left(\varepsilon_{5}\right)$ corrections are

$$
\begin{aligned}
\mathcal{R}_{1}= & -2 \mathcal{I}_{2} \sinh k \bar{h}-\frac{1}{C_{0}}\left(2 \mathcal{I}_{3} \operatorname{csch} 2 k \bar{h}+C_{1}\right) \\
\mathbf{R}_{2}= & \frac{C_{1}-\mathcal{U}}{C_{0}} C_{g \text { nvs }} \frac{\mathbf{k}}{k}+\mathcal{I}_{3} \mathbf{k} \bar{h} \operatorname{sech}^{2} k \bar{h} \\
& +\frac{\mathbf{k}}{k} C_{0}\left(2 \mathcal{I}_{1} \cosh k \bar{h}-\mathcal{I}_{2} \sinh k \bar{h}(2-\sinh k \bar{h})\right) \\
& +\tanh k \bar{h}\left(\mathbf{I}_{4}+\mathbf{I}_{5}-\frac{\mathbf{k}}{k} \mathcal{I}_{3}-\mathbf{k} \bar{h} C_{0} \mathcal{I}_{2}\right. \\
& \left.-\frac{\mathbf{k}}{k} C_{0} \mathcal{I}_{1} \sinh ^{2} k \bar{h}-\frac{1}{2 k} \mathbf{u}^{(0)}\right)
\end{aligned}
$$

which contain the terms,

$$
\begin{aligned}
C_{0} & =\sqrt{\frac{g}{k} \tanh k \bar{h}}, \quad \bar{h}=h+\eta^{(0)} \\
\mathcal{I}_{1}(z) & =\int_{-h}^{z} \frac{\mathcal{U}(\zeta)^{\prime \prime}}{k C_{0}} \sinh ^{2} k(\zeta+h) d \zeta \\
\mathcal{I}_{2}(z) & =\int_{-h}^{z} \frac{\mathcal{U}(\zeta)^{\prime \prime}}{2 k C_{0}} \sinh 2 k(\zeta+h) d \zeta \\
\mathcal{I}_{3}(z) & =\int_{-h}^{z} k \mathcal{U}(\zeta) \cosh 2 k(\zeta+h) d \zeta \\
\mathbf{I}_{4}(z) & =\int_{-h}^{z} 2 \mathbf{k} \mathcal{U}(\zeta) \sinh ^{2} k(\zeta+h) d \zeta \\
\mathbf{I}_{5}(z) & =\int_{-h}^{z} \frac{\mathbf{u}^{(0)^{\prime \prime}}}{2 k} \sinh ^{2} k(\zeta+h) d \zeta
\end{aligned}
$$

Note that the leading terms in the expressions for the wave action and its flux coincide with those for the case of no vertical shear (see Appendix D). This shows that the commonly-used WAE models based on Bretherton and Garrett [3] are actually the leading order approximations for currents with a vertical structure, and may be improved when the above approximation applies with no cost by using the value of the current at the surface rather than the depthaveraged velocity.

The main outcome of this paper can be summarised as follows: The WAE

$\frac{\partial I}{\partial t}+\nabla \cdot\left(\mathbf{C}_{\mathbf{g}} I\right)=0$

where its main terms are:

$$
\begin{aligned}
I & =\left\{\frac{a^{2} g}{\sigma}\left(1+\varepsilon_{5} \mathcal{R}_{1}\right)\right\}_{z=\eta^{(0)}} \\
\mathbf{C}_{\mathbf{g}} & =\left\{\left(C_{g_{\mathrm{nvs}}} \frac{\mathbf{k}}{k}+\mathbf{u}^{(0)}\right)\left(1-\varepsilon_{5} \mathcal{R}_{1}\right)+\varepsilon_{5} \mathbf{R}_{2}\right\}_{z=\eta}
\end{aligned}
$$

Here, the group velocity accurate to $O\left(\varepsilon_{5}\right)$ was easily found using a Taylor series on the division of Eq. (4.3) by Eq. (4.2). Eqs. (4.5)-(4.7) are the main findings of this paper; they provide an explicit WAE formulation for surface waves propagating in the presence of vertical shear, under assumptions (the same as in Skop [12]) of a weak current, gradient and curvature of the vertical structure of the current. We stress that even small discrepancies in the expressions for group velocities accumulate in the course of wave propagation, which, as we demonstrate below, might grow into significant discrepancy in wave amplitude predictions.

\section{Examples}

657

Here we consider an example of a characteristic current profile to examine the accuracy for local values of the wave action and group velocity provided by the new wave action formulation. A numerical code to solve exactly the problem and thus provide the reference has been written in Mathematica version 9.0.1.0. The Rayleigh equation has been solved with NDSolve with the Shooting Method. The resulting eigenfunction profile is used to calculate the exact values of the invariant and group velocity using Eqs. (3.11) and (3.13). We examine the discrepancies between the no-shear (Eqs. (D.7) and (D.10)) and the new approximation (Eqs. (4.2) and (4.7)) to the exact vertical shear formulation (Eqs. (3.11) and (3.13)).

An ambient current profile which has been shown to be typical of wind-induced currents in channels [38] is the wind-driven current at the surface and with an opposing current at larger depths, as shown in Fig. 1(a). This particular profile given by the was derived analytically [38] and tested numerically and experimentally for a steady, shear-induced turbulent flow typical of wind-induced currents. The analytical expression for this profile reads,

$U(z)=A u_{*} \ln \left[1+\frac{z}{z_{s}}\right]+B u_{*} \ln \left[1-\left(\frac{z}{z_{b}}+h\right)\right]$ 156

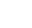
. .

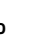

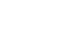
. , . . . . .

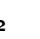

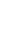


where $u_{*}$ is the surface friction velocity, $h$ is the water depth, $A=\frac{q_{2}}{p_{1} q_{2}-q_{1} p_{2}}, B=-\frac{q_{1}}{p_{1} q_{2}-q_{1} p_{2}}, p_{1}=$ $\gamma \frac{z_{s}}{h}, p_{2}=\gamma \frac{z_{s}}{z_{b}}, q_{1}=\left(1+\frac{z_{s}}{h}\right) \ln \left[1+\frac{h}{z_{s}}\right]-1, q_{2}=$ $\frac{z_{s}}{h} \ln \left[1+\frac{h}{z_{b}}\right]-1, z_{b}$ and $z_{s}$ are characteristic viscous sublayer thicknesses at the bottom and surface respectively and $\gamma$ is a constant characterising the intensity of the turbulence. We will denote the velocity at the surface as $U(0)$.

Fig. 1(a) shows the profile with the parameters $z_{s}=3.3 \times 10^{-4} h, z_{b}=1.0 \times 10^{-3} h, \gamma=1.0, k h=1$, $u_{*}=-1.0 \times 10^{-3} \mathrm{~ms}^{-1}$, which gives a top layer thickness $\delta_{s} \approx 0.34 h$ (marked with arrows) and the surface velocity $U(0)=1.0 \mathrm{~ms}^{-1}$. The parameters of the current were deliberately chosen to violate the smallness of gradient and curvature assumptions to show that the adopted approximations work very well far beyond the range of their asymptotic validity. The plot of the vertical velocity structure in Fig. 1(b) indeed shows that, for the approximation of taking into account the vertical shear, the vertical velocity $w_{0}+w_{1}$ is now in excellent agreement with the exact (numerical) solution. The discrepancy with the exact solution is less than $1 \%$, whereas the typically-used no vertical shear approximation gives an error of $10 \%$ for the same current profile.
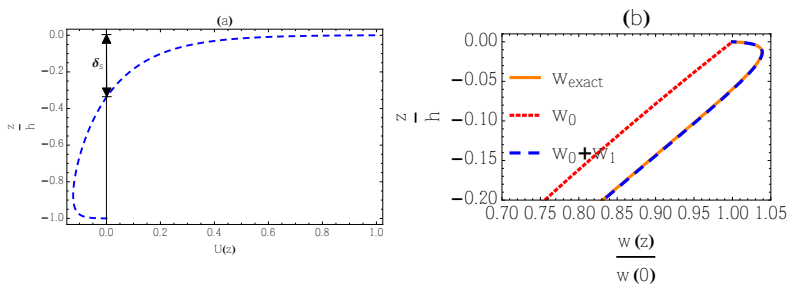

Figure 1: (a) Countercurrent flow profile derived by $\mathrm{Wu} \&$ Tsanis [38] defined in Eq. (5.1). (b) Comparison of the vertical velocity structure for the countercurrent profile for parameters $u_{*}=-1.0 \times 10^{-3}, z_{s}=3.3 \times 10^{-4} h, z_{b}=1.0 \times 10^{-3} h, \gamma=1.0$, $k h=1$ which gives a surface velocity of $u(0)=1.0 \mathrm{~ms}^{-1}$. The thickness of the upper layer is $\delta_{s}$ and the mean velocity in this upper layer is $U_{m}=0.143 \mathrm{~ms}^{-1}$.

The presented results demonstrate the substantial gain in accuracy provided by the approximate solutions compared to the widely used no-shear approximation. The no-shear group velocity $C_{\text {gnvs }}$ is specified by Eq. (D.10) in Appendix D. $\mathbf{u}^{(0)}$ is taken at the surface $z=\eta^{(0)}$, however we will also examine numerically whether this simple formulation shows an increase in accuracy over using a depthaveraged value of the current $U_{m}=1 / h \int_{-h}^{0} U(z) d z$. Figures 2, 3, 4 and F.8 show contours of \%-errors of (a) the approximated group velocity $C_{g}$, defined in Eq. (4.7), (b) our derived no-shear group velocity $C_{\text {gnvs }}$ defined in Eq. (D.10) with the surface value of the current $\mathbf{u}^{(0)}\left(\eta^{(0)}\right)$ and (c) the no-shear group velocity but now calculated with $U_{m}$, all compared to the exact group velocity $C_{\text {gvs }}$, which is calculated by dividing Eq. (3.13) by Eq. (3.11). The remaining panels are \%-errors of (d) the approximated invariant $I$, defined in Eq. (4.2) and (e) our derived no-shear invariant $I_{n v s}$, defined in Eq. (D.7) over the exact invariant $I_{v s}$ defined in Eq. (3.11). The current term $\mathbf{u}^{(0)}(z)$ does not appear in the expression for $I_{n v s}$.

The errors for the group velocity and the invariant are shown in Figs. 2(a)-(e) for co-propagating waves and ambient current. For very weak currents, $U / C_{0} \lesssim 0.02$ for all wavelengths, the errors for the group velocity are around $1 \%$ with this error increasing for stronger currents. This was to be expected since the approximation assumes a small current. Comparing Figs. 2(b) and (c) shows that by calculating the no-shear approximation with the surface value of the current velocity, rather than the depthaveraged current value, actually increases the errors over the exact solution for this particular vertical profile. A larger parameter space was studied than is shown here but the errors continue to grow with increasing current strength.
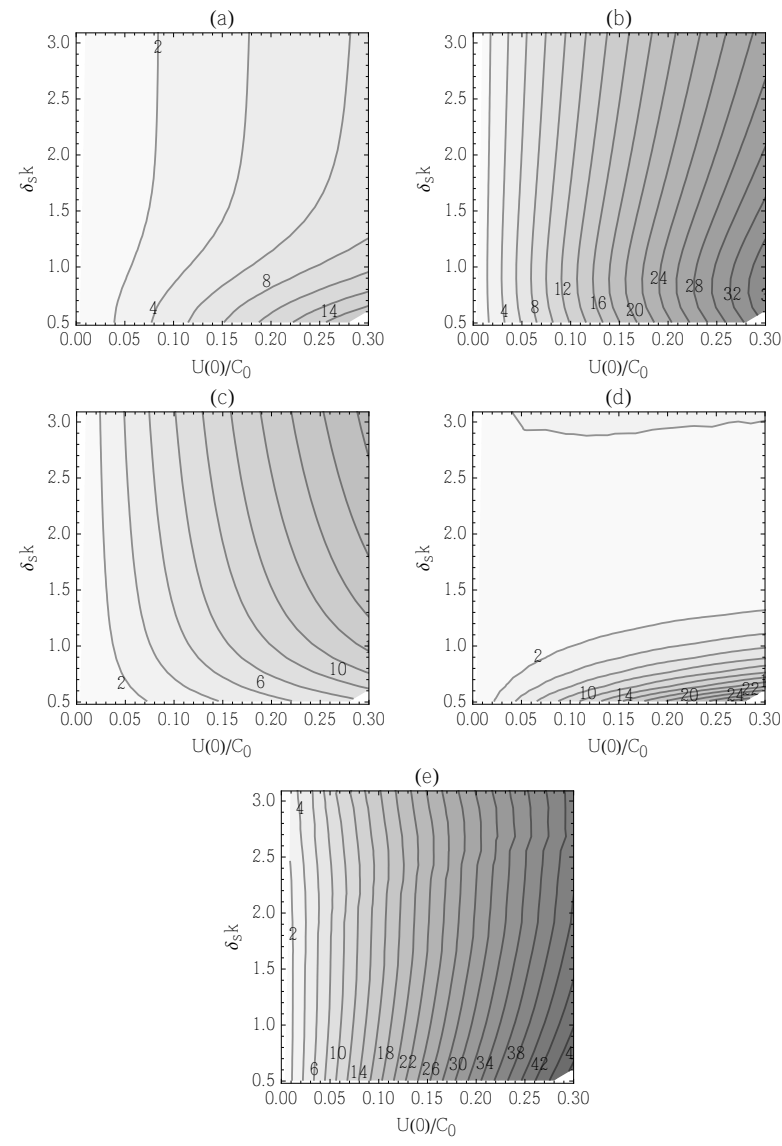

Figure 2: Wu \& Tsanis [38] countercurrent flow profile with copropagating waves. Contours of : (a) errors of the approximate group velocity to the exact group velocity, $C_{g} / C_{g v s}-1$ in $\%$, (b) the no-shear group velocity to the exact group velocity $C_{g_{n v s}} / C_{g v s}-1$ in \%, (c) the no-shear group velocity calculated with the depth-averaged velocity to the exact group velocity $\left(\left.C_{g_{n v s}}\right|_{U m}\right) / C_{g v s}-1$, (d) $I / I_{v s}-1$ and (e) $I_{n v s} / I_{v s}-1$, for parameters $z_{s}=2.2 \times 10^{-4} h, z_{b}=1.4 \times 10^{-4} h, \gamma=0.35$, $h=100 m$ and variation in $k$ and $u_{*} . U(0)$ is the velocity at the free surface. $\delta_{s}$ is the thickness of the upper layer $\approx 0.34 h$.

Figs. 3(d)-(e) show the errors for the invariant. Comparison of (d) and (e) clearly shows the gain accuracy provided by the adopted approximation compared to the no-shear one, especially for longer waves 

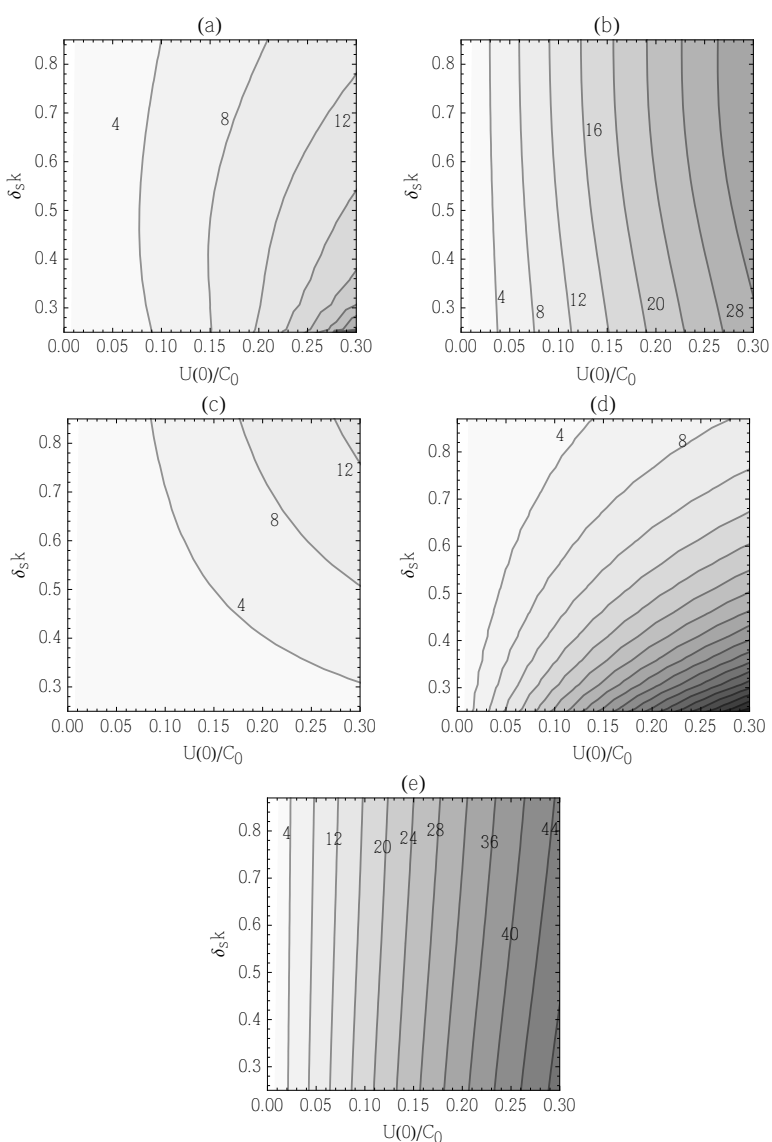

Figure 3: Wu \& Tsanis [38] countercurrent flow profile with co-propagating waves. Contours of (a) errors of the approximate group velocity to the exact group velocity $C_{g} / C_{g v s}-1$ in $\%$, (b) the no-shear group velocity to the exact group velocity $C_{g_{n v s}} / C_{g v s}-1$ in $\%$, (c) the no-shear group velocity calculated with the depth-averaged velocity to the exact group velocity $\left(\left.C_{g_{n v s}}\right|_{U m}\right) / C_{q v s}-1$ in $\%$, (d) $I / I_{v s}-1$ (in $\%$ ) and (e) $I_{n v s} / I_{v s}-1$ (in \%), for parameters $z_{s}=2.2 \times 10^{-4} h$, $z_{b}=1.4 \times 10^{-4} h, \gamma=0.35, h=20 \mathrm{~m}$ and variation in $k$ and $u_{*} . U(0)$ is the velocity at the free surface. $\delta_{s}$ is the thickness of the upper layer $\approx 0.34 h$.

where the error of the approximation almost disappears for all current strengths. Expectedly, the errors also increase for stronger currents.

When the waves are opposing the surface current, Fig. 4(a) shows that the errors for the group velocity with the adopted approximation are similar to those of the co-propagating waves in Fig. 3(a). However a comparison of Fig. 4(b) and Fig. 3(b) shows that the errors for the group velocity with the no-shear approximation are slightly larger for the waves are opposing the surface current than following it. The likely explanation of this asymmetry is that since our asymptotic expansion exploits smallness of current to wave celerity ratio, for the opposing current the effective phase velocity is a bit larger.

Another example, an idealized two-layer current profile, is examined in Appendix F. It should be noted that in this section, the focus of the examination was on local characteristics of the wave field such as the group velocity and the local adiabatic invariant. The examples of shear flows we analysed
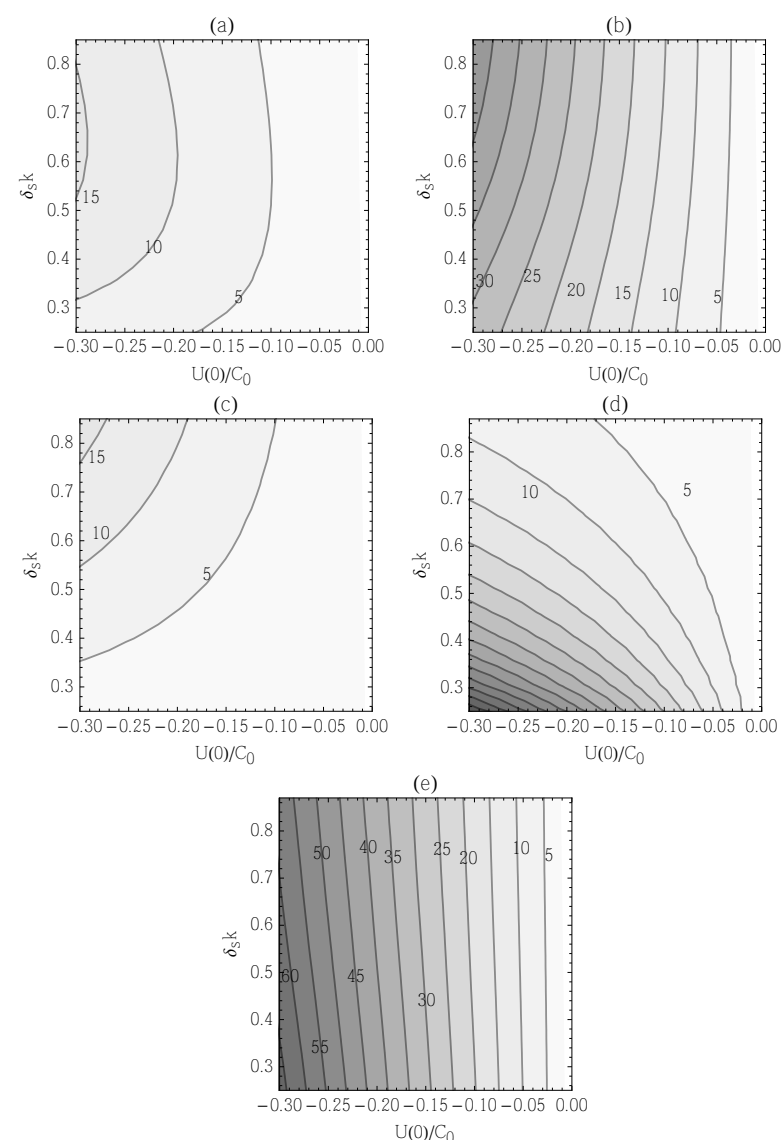

Figure 4: Wu \& Tsanis [38] countercurrent flow profile with opposing waves. Contours of (a) \%-errors of the approximated group velocity to the exact group velocity $C_{g} / C_{g v s}$, (b) the no-shear group velocity to the exact group velocity $C_{g_{n v s}} / C_{g v s}$, (c) the no-shear group velocity calculated with the depth-averaged velocity to the exact group velocity $\left(\left.C_{g_{n v s}}\right|_{U m}\right) / C_{g v s}$, (d) $I / I_{v s}$ and (e) $I_{n v s} / I_{v s}$, for parameters $z_{s}=2.2 \times 10^{-4} h, z_{b}=1.4 \times 10^{-4} h, \gamma=0.35, h=20 \mathrm{~m}$ and variation in $k$ and $u_{*} . U(0)$ is the velocity at the free surface. $\delta_{s}$ is the thickness of the upper layer $\approx 0.34 h$. The contours are at the same levels for each panel.

showed a noticeable gain in accuracy provided by the adopted approximation as compared to the no-shear approximation. Crucially, even small discrepancies can significantly affect the nonlocal properties of the solutions (e.g. envelope amplitude, ray trajectories, etc), which would cause the discrepancies to accumulate over distances and become much more significant. This is the subject of the next section where we show that the new formulation better predicts also these nonlocal properties with smaller errors from the exact solution.

\section{Wave rays and amplitudes}

Consider a model situation when both the current and wave field are steady in time and the horizontal current varies in one direction only, then Eq. (3.10) can be significantly simplified. By setting the $y$-axis as the lateral direction of no changes in the medium, the WAE takes the form

$\frac{\partial}{\partial x}\left(C_{g x} I\right)+C_{g y} \frac{\partial I}{\partial y}=0$ 
$831 \quad A(x)=\left[\frac{\mathrm{J}\left(x_{0}\right)}{\mathrm{J}(x)}\right]^{1 / 2} A\left(x_{0}\right)$

$837 \quad A(x)=\left[\frac{y^{\prime}\left(x_{0}\right)}{y^{\prime}(x)}\right]^{1 / 2} A\left(x_{0}\right)$.

$y(x)=\int_{x_{0}}^{x} \frac{C_{g y}}{C_{g x}} d \xi+s$, dition at $x=x_{0}$ as

$\mathcal{F}(y)=\left.C_{g x} I\right|_{x=x_{0}}$. determined from Eq. (D.12).

with the characteristics given in quadratures as

from the initial $y$-position $s$. The so far unspecified function $\mathcal{F}(\cdot)$ is then found using the boundary con-

When there is no lateral dependence in the boundary condition, $\mathcal{F}$ becomes a constant and the regular $1 \mathrm{D}$ solution of the WAE is retained. This applies for all three cases, the "exact" vertical shear, the new vertical shear approximation and the no-shear approximation, using of course the appropriate expression for the group velocity and invariant as detailed in $\S 3, \S 4$ and Appendix D respectively. The amplitude of the vertical velocity $A$ can be calculated from the solution of the WAE for the exact vertical shear formulation by dividing the solution by the wave action as defined in Eq. (3.11). From this, the wave elevation $a$ can be

Wave rays were studied in detail for a Gaussian surface current profile with no vertical shear by Mei et al. [39] although the group velocity and current velocity terms $u_{1}^{(0)}, u_{2}^{(0)}$ were omitted from the integrand. Three different scenarios were detailed where the ray either passed through the current after a deflection, it was reflected back by the current or became trapped inside the surface current [39]. Here, it was demonstrated that for a given set of parameters the exact and approximate shear and formulations allow the ray to pass through the current, while under the no shear formulation the hits the theoretical caustics. Thus, the no-shear formulation predicts a rough guess of the path only, which might dramatically differ from the true path captured well by the adopted vertical shear formulation. Within the framework of geometrical ray theory the amplitude of the refracted wave along the ray is determined [40] from

where $\mathrm{J}$ is the Jacobian of the transformation of rectangular coordinates to the ray coordinates. Here, it can be interpreted as being equivalent to the refraction index. For this particular case, which only has changes in the $x$-direction, this reduces to 


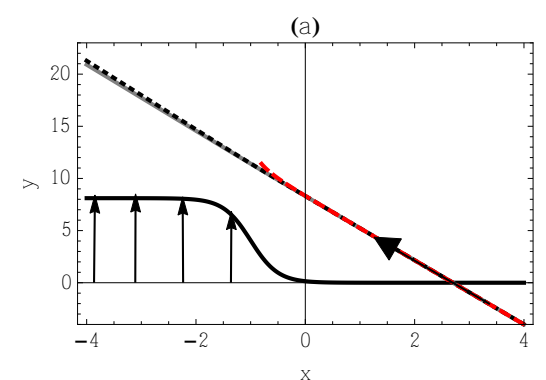

(b)

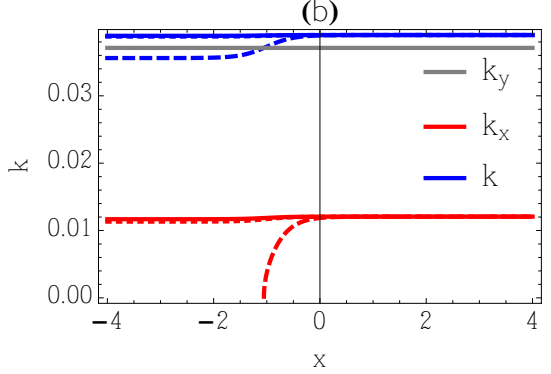

(c)

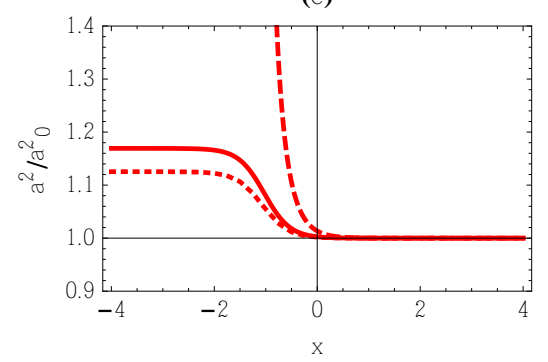

Figure 5: (a) Plan view sketch (solid black line with arrows, not to scale) of tanh current profile $(U(x, z)=-0.9(1+\tanh [2(x+$ 1)] $U(z)$ at the surface $z=0 . U(z)$ is the Wu and Tsanis vertical profile (see Eq. (5.1)) for a depth of $h=20 \mathrm{~m}$ with parameters $z_{s}=2.2 \times 10^{-4} h, z_{b}=1.4 \times 10^{-4} h, \gamma=0.35$, $u_{*}=0.09 \mathrm{~ms}^{-1}$ giving the maximum surface current $U(0) \approx$ $1.0 \mathrm{~ms}^{-1}$ and an depth averaged velocity, $U_{m}=0.126 \mathrm{~ms}^{-1}$, in the upper layer. Wave rays under different approximations for the same initial wave prescribed at $x=4$ : wavelength of $\approx 160 \mathrm{~m}$ uniform in $y$ incident obliquely (the angle $\theta=72^{\circ}$ to the $x$-axis. The approximations plotted: exact shear formulation (solid lines), no-shear approximation (dashed lines) and the adopted approximation (dotted lines). (b) Variations of the components of wavevector $\left(k_{x}, k_{y},|k|\right)$ across the horizontal plane. (c) the evolution of wave energy along the respective wave ray paths under different models of accounting shear (the same code as panel (a)). The wave is following the current.

isting wide separation of spatial and temporal scales between waves and currents we employ the standard WKB approximation. The derivation of the WAE follows V76 with the following new elements. We took into account the effect of Earth's rotation and examined its role, it has been found that for realistic assumptions on the values of rotation and wave periods, the Coriolis effect does not alter the WAE of Voronovich [4] (V76), although it does enter into the problem implicitly through the mean flow equations. In the process of derivation we also highlighted the junctions where taking into account the eddy viscosity, wind input and bottom friction (if necessary) would modify expression for the adiabatic invariant - the wave action.

The key difference with the V76 results is as fol-

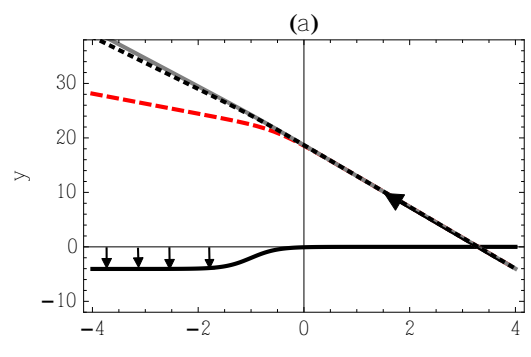

(b)

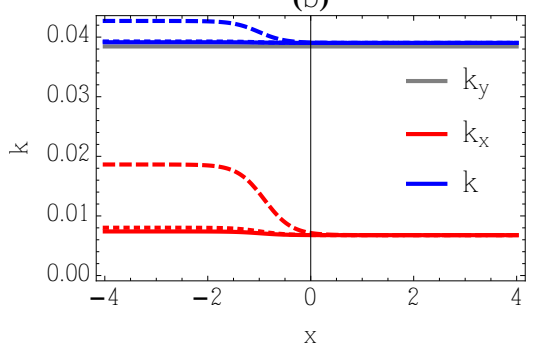

(c)

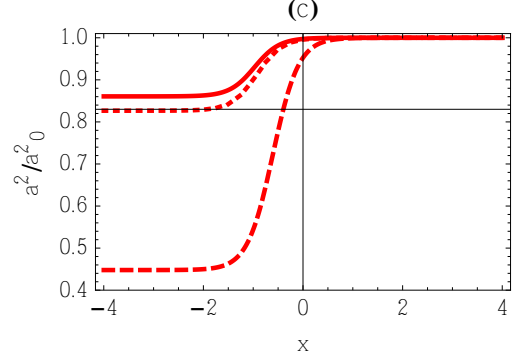

Figure 6: (a) Plan view sketch (solid black line with arrows, not to scale) of tanh current profile $(U(x, z)=0.9(1+$ $\tanh [2(x+1)]) U(z))$ at the surface $z=0 . \quad U(z)$ is the $\mathrm{Wu}$ and Tsanis vertical profile (see Eq. (5.1)) for a depth of $h=20 m$ with parameters $z_{s}=2.2 \times 10^{-4} h, z_{b}=1.4 \times 10^{-4} h$, $\gamma=0.35, u_{*}=0.09 \mathrm{~ms}^{-1}$ giving the maximum surface current $U(0) \approx-1.0 \mathrm{~ms}^{-1}$ and an depth averaged velocity, $U_{m}=-0.126 m s^{-1}$, in the upper layer. Wave rays under different approximations for the same initial wave prescribed at $x=4$ : wavelength of $\approx 160 \mathrm{~m}$ uniform in $y$ incident obliquely (the angle $\theta=80^{\circ}$ to the $x$-axis. The approximations plotted: exact shear formulation (solid lines), no-shear approximation (dashed lines) and the adopted approximation (dotted lines). (b) Variations of the components of wavevector $\left(k_{x}, k_{y},|k|\right)$ across the horizontal plane. (c) the evolution of wave energy along the respective wave ray paths under different models of accounting shear (the same code as panel (a)). The wave is opposing the current.

lows: the V76 expression for the wave action is implicit, it requires solving the Rayleigh boundary value problem for water waves at each point of the wave trajectory and calculating their cumulative effect over the depth. Here, we use explicit solutions to the boundary value problem provided by the leading order of an asymptotic expansion utilising smallness of the oceanic currents compared to wave phase velocity. The main conclusions of the work can be summarised as follows: (i) For dominant wind waves and swell and all conceivable currents the approximate formulae we put forward are providing good accuracy, which has been verified by direct comparison with the "exact" (under the WKBJ approximation) V76 results. (ii) The account of the vertical shear might lead to a significant departure in wave trajectories, wave lengths 
and amplitudes compared to the predictions of the currently employed "no shear" wave action model. Even for the situations where the underlying assumptions for the adopted approximation are violated, the formulae proved to be robust: there is a little loss of accuracy and a significant improvement for the group velocity and the invariant compared to the standard no-shear formulation of Bretherton and Garrett [3].

Implementation of this sheared-current approximation to existing wave models should be as simple as adjusting the expressions for the group velocity and the invariant. This should not lead to much higher computational costs since the weighted integrals of the vertical profile can be calculated at each point, while each node is independent and does not depend on neighboring nodes and therefore can be parallelized. Usually the starting point for the simulation are the data on incoming wave spectra at the outer boundary of the computational domain provided by either a buoy or a large scale wave forecasting model. The spectrum has to be discretized in the wavevector space and then for each Fourier harmonics the formulae, which we put forward, enable us to find the trajectory and evolution of wave parameters.

Here we very briefly discuss the range of the applicability of the results, then their implications and, finally, the perspectives of further studies. The main limitation on the WAE range of validity is due to the restrictive assumption of wave linear dynamics: the WAE describes phase averaged evolution of wave field until nonlinear interactions become essential, this interval is primarily determined by wave characteristic nonlinearity $\mu$ - the integral steepness of the wave spectrum. The waves propagating on a vertically sheared current are participating in two types of nonlinear resonant interactions: quartet interactions between the surface waves and triad interactions between a pair of surface waves and a vorticity wave supported by the inhomogeneity in the vertical distribution of vorticity (e.g., [41]). The characteristic timescale $T_{n l}$ of wave evolution due to quartet interactions is $\omega_{0}^{-1} \mu^{-4}$, where $\omega_{0}$ is the frequency of the wave of interest. The scale of validity of the WAE is restricted from above $\mathrm{T}$ _nl; it should be much smaller than $T_{n l}$, the time scale of the Hasselmann kinetic equation. It is the kinetic (rather than dynamic $\omega_{0}^{-1} \mu^{-2}$ ) timescale which is relevant here since both the WAE and the Hasselmann equation describe a phase-averaged evolution of wave field. Although the time scale of triad interactions is scaled as $\mu^{-2}$, the interaction coefficient has additional smallness due to the smallness of the current compared to wave celerity; this yields the time scale of triad interactions comparable or exceeding that of the standard four wave interactions (for details see [10]). For dominant wind waves the typical angular frequencies of the spectral peak, $\omega_{\text {peak }}$, are $\sim 1 \mathrm{rad} / \mathrm{s}$, while the characteristic steepness is $\approx 0.1$, this gives the estimate of the nonlinear time scale as $\sim 10^{4} s$. Hence, for wind waves $10^{3} s$ is a conservative estimate of the time scale of the WAE validity. For swell, the typical steepness is $\sim 5 \cdot 10^{-2}$ and characteristic periods are $\sim 10 \mathrm{~s}$, which gives the time scale of validity $\sim 10^{3} \mathrm{~s}$. A conversion of the time scales into the spatial scales depends on the specific bathymetry of the area under consideration, a rough estimate would be $\sim 10 \mathrm{~km}$, that is the scale of great interest in the context of coastal studies applications. Hence, there is a room for a nested small scale linear model based on the explicit WAE integrated with a local circulation model and, when possible, assimilating data on the shear currents provided by operational HF radars (e.g., [42]). There are numerous potential applications and implications of the explicit WAE coupled with the circulation model, here we mention just a 1002 few. As we already discussed the account of vertical ${ }_{1003}$ shear might result in order one effect in predicting 1004 the wave amplitude and wavelength. Employing the 1005 explicit WAE it is straightforward to find also the set- ${ }_{1006}$ up and set-down of wave field which are quadratic in 1007 amplitude, hence, the discrepancies with the no shear 1008 formulation will be even more pronounced. This is 1009 important for sediment transport, ship routing, vari- 1010 ous off shore activities.

There is a potential for further development of ${ }_{1012}$ the proposed WAE. First, although the employed 1013 explicit solution of the boundary value problem by ${ }^{1014}$ Skop [12] provides sufficient accuracy for the fore- ${ }^{1015}$ seeable applications, the experience of practical us- ${ }^{1016}$ age might require an improvement of the WAE accu- ${ }_{1017}$ racy, which can be achieved by taking the next or- ${ }_{1018}$ der in the asymptotic expansion using [37]. The 1019 error caused by adopting the WKBJ approximation 1020 is negligible everywhere except the narrow vicini- ${ }^{1021}$ ties of the caustics, even near the caustics integra- ${ }_{1022}$ tion over the spectrum in wavevector space will make ${ }_{1023}$ the caustics contribution insignificant since for each 1024 Fourier components the caustics is in a different lo- ${ }_{\mathbf{1 0 2 5}}$ cation. It might be possible to improve the accu- ${ }_{1026}$ racy of the WAE by taking into account simultane- ${ }_{1027}$ ously the quadratic nonlinearity responsible for triad ${ }_{\mathbf{1 0 2 8}}$ interactions with the vorticity modes and the eddy ${ }_{1029}$ viscosity: if the eddy viscosity is strong enough to ${ }_{1030}$ treat the vorticity waves as forced rather than free ${ }_{1031}$ modes, then it is in principle straightforward to in- ${ }_{1032}$ troduce a change of field variables which would elim- ${ }_{1033}$ inate quadratic nonlinearity similar to the procedure ${ }_{1034}$ employed for potential surface waves [43]. However, 1035 at present the eddy viscosity (or more sophisticated ${ }_{1036}$ description of the effect of subsurface turbulence) is ${ }_{1037}$ not reliably known yet, while the procedure being ${ }_{1038}$ straightforward in principle, is technically quite in- ${ }_{1039}$ volved. Hence it would be prudent to wait until the 1040 needed advance in understanding the effect of sub- ${ }_{1041}$ surface turbulence happens. The final destination of ${ }_{1042}$ the activity centered on the WAE is to combine the ${ }_{1043}$ account of vertical shear in the linear part of the equa- ${ }_{1044}$ tion (as it has been done in the present work) with ${ }_{1045}$ the nonlinear part of the kinetic equation (the colli- ${ }_{1046}$ sion integral). At present it is not clear how this goal ${ }_{1047}$ 
can be achieved, since there are irreconcilable conceptual difficulties: the collision integral operates in the wavevector space, while the linear WAE acts in the $\mathbf{x}$-space. This challenge requires deep thinking.

\section{Appendix A. Second-order equations}

On substituting Eq. (3.1) into Eqs. (2.5), (2.6) and retaining second-order terms in $a \varepsilon$ in the WKB expansion we obtain equations for the next order velocity terms,

$$
\begin{aligned}
\mathrm{i} \sigma \mathbf{u}^{(2)}- & w^{(2)} \frac{\partial \mathbf{u}^{(0)}}{\partial z}-\frac{\mathrm{ik}}{\rho_{0}} p^{(2)}=\mathbf{r}_{1} \\
\mathrm{i} \sigma w^{(2)}- & \frac{1}{\rho_{0}} \frac{\partial p^{(2)}}{\partial z}=r_{2} \\
\mathrm{ik} \cdot \mathbf{u}^{(2)}+ & \frac{\partial w^{(2)}}{\partial z}=-\nabla \cdot \mathbf{u}^{(1)} \\
\mathbf{r}_{1} \equiv & \frac{\partial \mathbf{u}^{(1)}}{\partial t}+\mathbf{u}_{i}^{(0)} \frac{\partial \mathbf{u}^{(1)}}{\partial x_{i}} \\
& +w^{(0)} \frac{\partial \mathbf{u}^{(1)}}{\partial z}+u_{i}^{(1)} \frac{\partial \mathbf{u}^{(0)}}{\partial x_{i}} \\
& +f \hat{z} \times \mathbf{u}^{(1)}+\frac{1}{\rho_{0}} \nabla p^{(1)} \\
r_{2} \equiv & \frac{d w^{(1)}}{d t}+w^{(1)} \frac{\partial w^{(0)}}{\partial z}
\end{aligned}
$$

Taking the vertical derivatives of Eqs. (A.1a) and (A.1c) together with Eq. (A.1b) yields a forced Rayleigh equation

$$
\begin{array}{r}
\frac{\partial^{2} w^{(2)}}{\partial z^{2}}-\left(k^{2}-\frac{\delta_{0}^{2}}{C-\mathcal{U}} \frac{\partial^{2} \mathcal{U}}{\partial z^{2}}\right) w^{(2)}=Q_{1} \\
Q_{1} \equiv-\frac{\mathbf{k}}{\sigma} \cdot \frac{\partial \mathbf{r}_{1}}{\partial z}+\frac{\mathrm{i} k^{2}}{\sigma} r_{2}
\end{array}
$$

In a manner similar to the analysis of first order equations, we express the pressure $p^{(2)}$ in terms of $w^{(2)}$ from Eqs. (A.1b) and (A.2)

$p^{(2)}=\frac{\mathrm{i} \rho_{0}}{k^{2}}\left(\mathbf{k} \cdot \mathbf{r}_{1}\right)+\frac{\mathrm{i} \rho_{0} \sigma}{k^{2}} \nabla \cdot \mathbf{u}^{(1)}+\frac{\mathrm{i} \rho_{0} \sigma^{2}}{k^{2}} \frac{\partial}{\partial z}\left(\frac{w^{(2)}}{\sigma}\right)$

The corresponding boundary conditions for the second-order equations are

$$
\begin{array}{rl}
\mathrm{i} \sigma \eta^{(2)}+w^{(2)}=r_{3} & z=\eta^{(0)} \\
p^{(2)}=\rho_{0} g \eta^{(2)} & z=\eta^{(0)} \\
w^{(2)}=Q_{2} & z=-h .
\end{array}
$$

where

$$
\begin{aligned}
Q_{2} \equiv & -\mathbf{u}^{(1)} \cdot \nabla h \\
r_{3} \equiv & \frac{d \eta^{(1)}}{d t}+\mathbf{u}^{(1)} \cdot \nabla \eta^{(0)} \\
& +\left(\frac{\partial \mathbf{u}^{(0)}}{\partial z} \cdot \nabla \eta^{(0)}-\frac{\partial w^{(0)}}{\partial z}\right) \eta^{(1)}
\end{aligned}
$$

Using Eqs. (A.3), (A.4a) and (A.4b) we derive the following free surface boundary condition for $w_{2}$

$$
{ }_{1084} \frac{\partial w^{(2)}}{\partial z}+\left(\frac{\delta_{0}}{(C-\mathcal{U})} \frac{\partial \mathcal{U}}{\partial z}-\frac{g}{(C-\mathcal{U})^{2}}\right) w^{(2)}=Q_{3}(\mathrm{~A} .6)
$$

on $z=\eta^{(0)}$, where

$Q_{3} \equiv-\frac{\mathbf{k}}{\sigma} \cdot \mathbf{r}_{1}-\frac{g k^{2}}{\sigma^{2}} r_{3}-\nabla \cdot \mathbf{u}^{(1)}$.

By virtue of (3.7a) $r_{3}$ can be written in terms of the ${ }^{1087}$ first-order vertical velocity

$$
\begin{aligned}
r_{3}= & i \frac{d}{d t}\left(\frac{w^{(1)}}{\sigma}\right)+\mathbf{u} \cdot \nabla \eta^{(0)} \\
& +\frac{i w^{(1)}}{\sigma}\left(\frac{\partial \mathbf{u}^{(0)}}{\partial z} \cdot \nabla \eta^{(0)}-\frac{\partial w^{(0)}}{\partial z}\right) .
\end{aligned}
$$

\section{Appendix B. The solvability condition for the ${ }_{1091}$ system

The solvability condition of the inhomoge- 1093 neous linear boundary-value problem given by 1094 Eqs. (A.2), (A.4c) and (A.6) allows for the construc- 1095 tion of the wave action conservation equation by fol- 1096 lowing the general derivation for second order ordi- 1097 nary differential equations [see 44, section 15.4] (al- 1098 beit with a different family of boundary conditions 1099 where here, $\Delta_{12} \neq 0$ rather than $\Delta_{13} \neq 0$, in their ${ }_{1100}$ notation). For brevity $w^{(2)} \equiv w_{2}$ the equation system ${ }_{1101}$ is written as

$w_{2}^{\prime \prime}+\Gamma w_{2}=Q_{1}, \quad-h<z<\eta_{0}$,

$$
w_{2}=Q_{2}, \quad z=-h,
$$

$w_{2}^{\prime}+\Lambda w_{2}=Q_{3}, \quad z=\eta^{(0)}$,

where

$\Gamma=-k^{2}+\frac{\delta_{0}^{2}}{C-\mathcal{U}} \frac{\partial^{2} \mathcal{U}}{\partial z^{2}}$,

$\Lambda=\frac{\delta_{0}}{(C-\mathcal{U})} \frac{\partial \mathcal{U}}{\partial z}-\frac{g}{(C-\mathcal{U})^{2}}$.

The adjoint system and the solvability equation 1109 can be found by multiplying Eq. (B.1a) by the ad- ${ }_{1110}$ joint function $w^{*}$ and integrating from $z=-h$ to 1111 $z=\eta^{(0)}$,

$\int_{-h}^{\eta^{(0)}}\left(w_{2}^{\prime \prime}+\Gamma w_{2}\right) w^{*} d z=\int_{-h}^{\eta^{(0)}} Q_{1} w^{*} d z$

(B.3) ${ }^{1113}$

Integrating by parts this equation is given by [see $44,{ }_{1114}$ equation 15.73]

$$
\begin{gathered}
\int_{-h}^{\eta^{(0)}}\left(w^{* \prime \prime}+\Gamma w^{*}\right) w_{2} d z+\left[w^{*} w_{2}^{\prime}-w^{* \prime} w_{2}\right]_{-h}^{\eta_{0}} \\
=\int_{-h}^{\eta^{(0)}} Q_{1} w^{*} d z .
\end{gathered}
$$

The homogeneous equation of the adjoint system is 1118 written by setting the integrand of the left hand side 1119 of equation (B.4) to zero. This shows that it coincides 1120 with the homogeneous part of Eqs. (A.2)

$w^{* \prime \prime}+\Gamma w^{*}=0, \quad-h<z<\eta^{(0)}$.

By setting $Q_{1,2,3}=0$, the boundary conditions of the ${ }_{1123}$ adjoint system are be defined from (B.4), 1124

$\left[w^{*} w_{2}^{\prime}-w^{* \prime} w_{2}\right]_{\eta_{0}}-\left[w^{*} w_{2}^{\prime}-w^{* \prime} w_{2}\right]_{-h}=0$ 
and by substituting Eqs. (B.1b) and (B.1c) to Eq. (B.6) results in

$$
\begin{aligned}
w^{*} & =0, & & z=-h, \\
w^{* \prime}+\Lambda w^{*} & =0, & & z=\eta^{(0)} .
\end{aligned}
$$

By inspecting the homogeneous boundary value problem given in Eqs. (3.6), (3.7c) and (3.8), it is apparent that these two systems are self-adjoint systems, and $w^{(1)}$ is the adjoint solution of the inhomogeneous boundary-value problem. Hence, $w^{(1)}$ can be substituted for $w^{*}$, i.e.

$w^{*}=w^{(1)}$.

The solvability condition is formulated upon substitution of Eqs. (B.1), (B.5), (B.7) and (B.8) into Eq. (B.4), which yields

$\int_{-h}^{\eta^{(0)}} Q_{1} w^{(1)} d z-\left.w^{(1)} Q_{3}\right|_{z=\eta^{(0)}}-\left.Q_{2} \frac{\partial w^{(1)}}{\partial z}\right|_{z=-h}=0$.

This condition can be reduced to a conservation law under the following steps. Substitute back in the expressions for $Q_{1}, Q_{2}$ and $Q_{3}$ from Eqs. (A.2b), (A.5a) and (A.7) respectively, multiply by $\mathrm{i} / k^{2}$ and then collect all the terms that contain a time derivative which will duly define the adiabatic invariant,

$$
\begin{aligned}
T_{1}=\int_{-h}^{\eta^{(0)}} \frac{\mathrm{i}}{k^{2}}\left(-\frac{\mathbf{k}}{\sigma} \cdot \frac{\partial^{2} \mathbf{u}^{(1)}}{\partial z \partial t}+\frac{i k^{2}}{\sigma} \frac{\partial w^{(1)}}{\partial t}\right) w^{(1)} d z \\
+\left[\frac{i g}{\sigma^{2}} w^{(1)} \frac{\partial \eta^{(1)}}{\partial t}+\frac{i w^{(1)}}{\sigma k^{2}} \mathbf{k} \cdot \frac{\partial \mathbf{u}^{(\mathbf{1})}}{\partial t}\right]_{z=\eta^{(0)}}
\end{aligned}
$$

From the continuity equation, $i \mathbf{k} \cdot \mathbf{u}^{(1)}=-\partial w^{(1)} / \partial z$ and by using the horizontal and vertical momentum fluxes of the mean flow $T_{1}$ reduces to the adiabatic invariant $I$ and the solvability condition, Eq. (B.9) reduces to

$$
\frac{\partial I}{\partial t}+\nabla \cdot\left(\mathbf{C}_{\mathbf{g}} I\right)=0
$$

where

$$
\begin{aligned}
I=- & \int_{-h}^{\eta^{(0)}} \frac{1}{2 \sigma^{2} k^{2}} \frac{\partial^{2} \sigma}{\partial z^{2}} w^{2} d z \\
& +\left[\left(\frac{g}{\sigma^{3}}+\frac{1}{2 \sigma^{2} k^{2}} \frac{\partial \sigma}{\partial z}\right) w^{2}\right]_{z=\eta^{(0)}}
\end{aligned}
$$

$$
\mathbf{C}_{\mathbf{g}} I=-\int_{-h}^{\eta^{(0)}}\left\{\frac{1}{2 \sigma^{2} k^{2}} \frac{\partial^{2} \sigma}{\partial z^{2}} \mathbf{u}^{(0)}\right.
$$$$
\left.-\frac{1}{2 \sigma k^{2}} \frac{\partial^{2} \mathbf{u}^{(0)}}{\partial z^{2}}+\frac{\mathbf{k}}{k^{2}}\right\} w^{2} d z
$$$$
+\left[\left(\left(\frac{g}{\sigma^{3}}+\frac{1}{2 \sigma^{2} k^{2}} \frac{\partial \sigma}{\partial z}\right) \mathbf{u}^{(0)}\right.\right.
$$$$
\left.\left.-\frac{1}{2 \sigma k^{2}} \frac{\partial \mathbf{u}^{(0)}}{\partial z}+\frac{g \mathbf{k}}{\sigma^{2} k^{2}}\right) w^{2}\right]_{z=\eta^{(0)}}
$$

and the superscript 1 has now been dropped from the $\mathrm{w}$ for clarity of notation. Dividing Eq. (3.13) by Eq. (3.11) gives an expression for the group ve- ${ }_{1165}$ locity. In the absence of the vertical derivatives, it 1160 is clear that Eqs. (3.11) and (3.13) reduce to the ${ }_{1167}$ usual expressions for the wave action invariant and 1168 its product with the wave group velocity. These are 1169 the expressions which are used in the spatial and time ${ }_{\mathbf{1 1 7 0}}$ discretization of wave models. To improve the wave 1171 models to take into account the vertical variability of ${ }_{1172}$ the flow, these extra vertical derivative terms must be ${ }^{1173}$ added to the models. In addition, it can be seen that ${ }_{1174}$ the inclusion of Earth's rotation $f$, to the flow did not $\quad{ }_{1175}$ change the wave action equation of Voronovich [4]. $\quad{ }_{1176}$

\section{Appendix C. The solution of the homoge- ${ }_{1177}$ neous Rayleigh equation

Since the current $\mathcal{U}(z)$ is now a function of the ver- 1179 tical coordinate, the group velocity cannot be defined ${ }_{1180}$ a priori which is obviously problematic for the WAE ${ }_{1181}$ which is solved for wave models. Consequently, the ${ }_{1182}$ perturbation method of Stewart \& Joy [11], Skop [12] ${ }_{1183}$ and Kirby \& Chen [37] can be employed, in a similar ${ }_{1184}$ manner to previous literature [13] to determine vari- ${ }_{1185}$ ous approximations to the dispersion relation $\sigma$ and ${ }_{1186}$ the vertical velocity $w$ so that analytical perturbation $\quad{ }_{1187}$ solutions can be used in the WAE.

By assumption of small currents, $\mathcal{U}(z)=\varepsilon_{4} \mathcal{U}(z),{ }_{1189}$ small current gradients $\mathcal{U}^{\prime}(z)=\varepsilon_{4} \mathcal{U}^{\prime}(z)$, and small 1190 current vertical curvature, $\mathcal{U}^{\prime \prime}(z)=\varepsilon_{4} \mathcal{U}^{\prime \prime}(z), \delta_{0}^{2} \leq{ }_{1191}$ $O(1)$, Eq. (3.6) can be ordered with the small param- ${ }_{1192}$ eter $\varepsilon_{4}$ as

$$
\begin{array}{r}
O\left(\frac{\delta_{0}^{2}}{C-\mathcal{U}} \frac{\partial^{2} \mathcal{U}}{\partial z^{2}}\right)=O\left(\delta_{0}^{2} \frac{\partial^{2}}{\partial z^{2}}\left(\frac{\mathcal{U}}{C}\right)\right) \\
=O\left(\frac{\delta_{0}}{C-\mathcal{U}} \frac{\partial \mathcal{U}}{\partial z}\right) \sim \varepsilon_{4} \ll 1 .
\end{array}
$$

By inserting the perturbation series in Eqs. (4.1) 1196 into the Rayleigh Eq. (3.6) and boundary conditions ${ }_{1197}$ $(3.7 \mathrm{c})$ and (3.8), equations to various orders in $\varepsilon_{4}$ are ${ }_{1198}$ obtained, which can subsequently be solved for the 1199 corresponding $C$ and $w$.

\section{Appendix C.1. Solution of the zeroth-order} To $O\left(\varepsilon^{0}\right)$ the zeroth-order equations and boundary 1202 conditions are:

$$
\begin{aligned}
& C_{0}\left(\frac{\partial^{2} w_{0}}{\partial z^{2}}-k^{2} w_{0}\right)=0, \quad-h \leq z \leq \eta^{(0)} \quad \text { (C.1a) }{ }^{1204} \\
& C_{0}^{2} \frac{\partial w_{0}}{\partial z}-g w_{0}=0, \quad z=\eta^{(0)} \quad \text { (C.1b) }{ }_{1205} \\
& w_{0}=0, \quad z=-h . \quad \text { (C.1c) } 1206
\end{aligned}
$$

Solving the zeroth-order Rayleigh Eq. (C.1a) with the 1207 bottom boundary condition (C.1c) yields $\quad 1208$ $w_{0}=A\left(\varepsilon_{1} \mathbf{x}\right) \sinh k(z+h)$.

Substituting (C.2) to the surface boundary condition 1210 results in

1211

$C_{0}=\sqrt{\frac{g}{k} \tanh k \bar{h}}$

(C.3) ${ }_{1212}$ 
with $\bar{h}=h+\eta^{(0)}, \mathcal{U}\left(\eta^{(0)}\right)$ and $\mathcal{U}^{\prime}\left(\eta^{(0)}\right)$ defined as $\mathcal{U}\left(\varepsilon_{1} \mathbf{x}, \eta^{(0)}\right)$ and $\partial \mathcal{U}\left(\varepsilon_{1} \mathbf{x}, z\right) /\left.\partial z\right|_{z=\eta^{(0)}}$ respectively. Using definitions (3.4) and (3.5), Eq. (C.3) enables us also to find the zeroth-order Doppler shifted frequency as

$$
\sigma_{0}=k\left(C_{0}-\mathcal{U}\right)=\sqrt{g k \tanh k \bar{h}}-\mathbf{k} \cdot \mathbf{u}^{(0)}
$$

which is equivalent to the known linear solution for waves on a depth-independent current.

\section{Appendix C.2. Solution of the first-order}

To $O\left(\varepsilon^{1}\right)$ the first-order equations and boundary conditions are:

$$
\begin{array}{rlrl}
\frac{\partial^{2} w_{1}}{\partial z^{2}} & -k^{2} w_{1}=-\frac{\left(C_{1}-\mathcal{U}\right)}{C_{0}} \frac{\partial^{2} w_{0}}{\partial z^{2}} & \\
& +\left(\frac{k^{2}\left(C_{1}-\mathcal{U}\right)}{C_{0}}+\frac{\delta_{0}^{2}}{C_{0}} \frac{\partial^{2} \mathcal{U}}{\partial z^{2}}\right) w_{0} & \text { (C.5a) } \\
C_{0}^{2} \frac{\partial w_{1}}{\partial z} & +C_{0} \frac{\partial \mathcal{U}}{\partial z} w_{0}-g w_{1} & \\
& +2 C_{0}\left(C_{1}-\mathcal{U}\right) \frac{\partial w_{0}}{\partial z}=0, & z=\eta^{(0)}(\mathrm{C} .5 \mathrm{~b}) \\
w_{1} & =0, \quad z=-h .(\mathrm{C} .5 \mathrm{c})
\end{array}
$$

The homogeneous solution for Eq. (C.5a) with the bottom boundary condition (C.5c) yields the same solution as the zeroth-order problem and can therefore be neglected. The particular solution can be found using the variation of parameters method, which results in

$w_{1}=A\left(\cosh k(h+z) \mathcal{I}_{1}(z)-\sinh k(h+z) \mathcal{I}_{2}(z)\right)($ C.6 $)$ where $\mathcal{I}_{1}$ and $\mathcal{I}_{2}$ are defined in Eq. (4.4). Here $\mathcal{U}$ and $\mathcal{U}^{\prime \prime}$ in the integrands are defined as $\mathcal{U}\left(\varepsilon_{1} \mathrm{x}, \zeta\right)$ and $\partial^{2} \mathcal{U}\left(\varepsilon_{1} \mathbf{x}, \zeta\right) / \partial \zeta^{2}$ respectively. Eq. (C.6) can be now substituted to Eq. (C.5b) to produce the first-order correction to the wave celerity as

$$
\left.\left.C_{1}=\frac{C_{0}}{\sinh 2 k \bar{h}} I_{1}\left(\eta^{(0)}\right)+\mathcal{U}\left(\eta^{(0)}\right)\right)-\frac{C_{0}^{2}}{2 g} \mathcal{U}^{\prime}\left(\eta^{(0)}\right)\right)
$$

The first-order Doppler shifted frequency is given simply as

$\sigma_{1}=k C_{1}$.

\section{Appendix D. Wave Action Equation for cur- rents with no vertical shear}

Currents with no vertical shear imply that all the vertical derivatives in Eqs. (3.11) and (3.13) are set to zero and the conservation law in the case of no vertical shear takes the form

$\frac{\partial I_{\mathbf{n v s}}}{\partial t}+\nabla \cdot\left(\mathbf{C}_{\mathbf{g}} I_{\mathbf{n v s}}\right)=0$

where

$$
I_{\mathrm{nvs}}=\left[\frac{g}{\sigma^{3}} w^{2}\right]_{z=\eta^{(0)}}
$$

$$
\begin{aligned}
\mathbf{C}_{\mathbf{g}} I_{\mathrm{nvs}}= & -\int_{-h}^{\eta_{0}} \frac{\mathbf{k}}{k^{2}} w^{2} d z \\
& +\left[\left(\frac{g}{\sigma^{3}} \mathbf{u}^{(0)}+\frac{g \mathbf{k}}{\sigma^{2} k^{2}}\right) w^{2}\right]_{z=\eta^{(0)}}
\end{aligned}
$$

The solution for the Rayleigh Eq. (3.6) with the ${ }^{1257}$ boundary conditions (3.7c) and (3.8) without vertical ${ }_{1258}$ shear takes the form

$$
\begin{aligned}
w_{\mathrm{nvs}} & =A\left(\varepsilon_{1} \mathbf{x}\right) \frac{\sinh k(z+h)}{\sinh k \bar{h}} \\
C_{\mathrm{nvs}} & =\frac{1}{k} \sqrt{g k \tanh k \bar{h}} \\
\sigma_{\mathrm{nvs}} & =\sqrt{g k \tanh k \bar{h}} .
\end{aligned}
$$

It can be easily seen that for no-vertical-shear con- ${ }_{1263}$ ditions the perturbation solution presented in $\S$ Ap- ${ }_{1264}$ pendix $\mathrm{C}$ degenerates to the above accurate solution. 1265 Substituting Eqs. (D.4) and (D.5) into Eqs. (D.2) and 1266 (D.3) yields

$$
\begin{aligned}
I_{\mathrm{nvs}}= & A^{2}\left\{\frac{g}{\sigma^{3}}\right\}_{z=\eta^{(0)}} \\
\mathbf{C}_{\mathbf{g}} I_{\mathrm{nvs}}= & A^{2}\left\{\frac { g } { \sigma ^ { 3 } } \left[\frac{1}{2} C_{d \mathrm{nvs}}\left(1+\frac{2 k \bar{h}}{\sinh 2 k \bar{h}}\right) \frac{\mathbf{k}}{k}\right.\right. \\
& \left.\left.+\mathbf{u}^{(0)}\right]\right\}_{z=\eta^{(0)}} .{ }^{1269} \\
& \text { (D.8) }{ }^{1270}
\end{aligned}
$$

Upon division by $g$, the conservation law takes the ${ }^{1271}$ form

$\frac{\partial}{\partial t}\left[\frac{A^{2}}{\sigma^{3}}\right]_{z=\eta^{(0)}}+\nabla \cdot\left[\left(C_{g \mathrm{nvs}} \frac{\mathbf{k}}{k}+\mathbf{u}^{(0)}\right) \frac{A^{2}}{\sigma^{3}}\right]_{z=\eta^{(0)}}=0$

where 1274

$C_{g \mathrm{nvs}}=\frac{1}{2} C_{d \mathrm{nvs}}\left(1+\frac{2 k \bar{h}}{\sinh 2 k \bar{h}}\right)+\mathbf{u}^{(0)}(z)$.

Furthermore, multiplying Eq. (3.7a) by its complex 1276 conjugate 1277

$\left.|\eta|^{2} \sigma^{2}\right|_{z=\eta^{(0)}}=\left.w^{2}\right|_{z=\eta^{(0)}}$,

yields the relation between the amplitude of the sur- ${ }_{\mathbf{1 2 7 9}}$ face elevation $(a)$ and the amplitude of the vertical ${ }_{1280}$ velocity $(A)$ :

1281

$A^{2}=\left.a^{2} \sigma^{2}\right|_{z=\eta^{(0)}}$.

Substituting Eq. (D.12) to Eq. (D.9) allows writing ${ }_{1283}$ the conservation law using the common surface ele- ${ }_{\mathbf{1 2 8 4}}$ vation amplitude wave action formulation 1285 $\frac{\partial}{\partial t} \mathcal{N}_{\text {nvs }}+\nabla \cdot\left\{\left[C_{g \text { nvs }} \frac{\mathbf{k}}{k}+\mathbf{u}^{(0)}\right]_{z=\eta^{(0)}}^{\mathcal{N}_{\text {nvs }}}\right\}=0\left(\right.$ D.13) ${ }_{1286}$ with the wave action defined as

$\mathcal{N}_{\mathrm{nvs}}=\left[\frac{a^{2}}{\sigma}\right]_{z=\eta^{(0)}}$.

\section{Appendix E. Linear vertical shear current ${ }_{1289}$ profile 1290}

Assuming a linear vertical current profile provides 1291 an exact analytical solution. Take a profile of the 1292 form $U(z)=p z+q$ where $p$ and $q$ are constants, 1293 the gradient $U^{\prime}(z)=p$ and curvature $U^{\prime \prime}(z)=0 . \quad{ }_{1294}$ The solution to the Rayleigh Eq. (3.6) is the same as 1295 
the case for no-shear given by Eq. (D.4). The wave dispersion becomes,

$\Omega=k q \pm \sqrt{g k \tanh h k}-\frac{1}{2} p \tanh h k(1 \mp 1)$.

Substitution of the velocity, dispersion and their derivatives into the WAE defined in Eqs. (3.11) and (3.13), gives exact analytical expressions for the invariant and group velocity

$$
\begin{aligned}
I & =a^{2}\left[\frac{g}{\sigma_{s}}-\frac{p}{2 k}\right]_{z=\eta^{(0)}} \\
\mathbf{C}_{\mathbf{g}} & =\left[p \eta^{(0)}+q+\frac{2 \mathbf{k} \sigma\left(g-\left(h+\eta^{(0)}\right) \sigma^{2}\right)-p \sigma^{2}}{k(2 g k-p \sigma)}\right]
\end{aligned}
$$

where Eq. (E.3) is equivalent to that of Jonsson et al. [19].

\section{Appendix F. Two-layer current profile}

An idealized analytical velocity profile describing situations common in the upper ocean and in estuaries when there is a density jump, is a two-layer profile in which the top layer has a constant velocity and the bottom layer has zero velocity, such as that shown in Fig. F.7(a) where the current velocity profile is defined as

$U(z)=m(1+\tanh [n(z+b)])$

where $b$ is the depth of the upper layer and $n$ determines the sharpness of the transition between layers and $m$ determines the magnitude of the velocity at the surface. The profile is often used for the modeling of mixing in stratified flows [45]. We will use this model with deliberately chosen very sharp transition to demonstrate that the adopted approximation works well even when assumptions of smallness of gradients and curvature are violated.

Fig. F.7(a) is the profile with the parameters $k h=$ $2.5, U=2.0 \mathrm{~ms}^{-1}$ in the top layer and zero in the lower layer, $b=0.2 h, m=1, n=20$. Again, even for this extremely simplified profile, the vertical velocity structure provided by the adopted approximation is greatly improved, as is evident in Fig. F.7(b). It can be seen that the $w_{0}+w_{1}$ approximation is much closer to the exact vertical structure: the errors are reduced to within $4 \%$ whereas for the $w_{0}$ term only, the maximum error is about $10 \%$.

Results shown in Fig. F.8 are errors for the group velocity and the invariant for a variation of wavelength and current velocity and a fixed $b / h=0.1$. Fig. F.8(a) shows that for increasing $U / C_{0}$, the errors to the group velocity from the new approximation over the exact vertical shear approximation are typically half in comparison to the no-shear formulation in Fig. F.8(b) for small values of $k b$. For $U / C_{0}<0.1$, the errors are typically below $5 \%$ for longer waves. Fig. F.8(b) shows that using the surface value of the
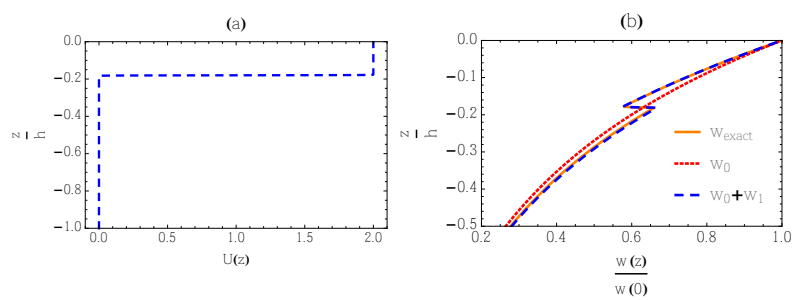

Figure F.7: (a) Two-layer profile $U(z)=m(1+\tanh [n(z+b)])$ where $b$ is the depth of the upper layer and $n$ determines the sharpness of the transition between the layers and $m$ specifies the magnitude of the velocity at the surface. (b) comparison of vertical velocity structure for the two-layer profile, $k h=2.5$, $U=2.0 \mathrm{~ms}^{-1}, b=0.2 h, m=1, n=20$.

current for the no shear approximation is more accu- $\mathbf{1 3 4 5}$ rate than using the value of the depth-averaged cur- 1346 rent velocity as shown in Fig. F.8(c).

For all wavelengths and a weak current, the ap- 1348 proximation to the invariant $I$ is excellent, but also 1349 for shorter waves on a relatively strong current, as $\mathbf{1 3 5 0}$ evident from Fig. F.8(d). The errors do become large ${ }_{1351}$ however for long waves on a thin top layer and a 1352 strong current. This is an improvement over the no- ${ }_{1353}$ shear formulation in Fig. F.8(e) which gives sizable ${ }_{1354}$ errors at all wave numbers for all current strengths. $\mathbf{1 3 5 5}$ Fig. F.8(f) shows the error for the invariant for the ${ }_{\mathbf{1 3 5 6}}$ no-shear formulation but calculated with the mean 1357 velocity. The no-shear expression for the invariant, ${ }_{1358}$ Eq. (D.7) does not contain the velocity so the no- 1359 shear invariant will be the same regardless of whether ${ }_{1360}$ the surface or mean velocity value is used.

For a fixed value of a strong current $\left(U \sim{ }_{1362}\right.$ $1.5 \mathrm{~ms}^{-1}$ ), Fig. F.9(a) shows that under the new ${ }_{1363}$ adopted approximation, for thin layers the errors for ${ }_{\mathbf{1 3 6 4}}$ the group velocity are reduced to about $10 \%$, which 1365 is noticeably better than the no-shear formulation 1366 shown in Fig. F.9(b). As expected, both formula- ${ }_{1367}$ tions tend to the exact solution as the thickness of ${ }_{1368}$ the top layer tends to $h$ as evident in Figs. F.9(a) ${ }_{1369}$ and (b). Again the no-shear formulation works bet- 1370 ter in most of the parameter space when the surface ${ }_{1371}$ value of the current is used rather than the mean $\mathbf{1 3 7 2}$ value as evident in Figs. F.9(b) and (c). For the in- ${ }_{1373}$ variant, Figs. F.9(d) and (e) show that in nearly all ${ }_{\mathbf{1 3 7 4}}$ of the parameter space, the adopted approximation is $\mathbf{1 3 7 5}$ very accurate. There is a small region of the parame- ${ }_{1376}$ ter space, for thin top layers and medium to shallow $1 \mathbf{1 3 7 7}$ water, $k h<2$, for which there are some errors com- 1378 pared to more widely-distributed errors in the tradi- ${ }_{1379}$ tional no-shear formulation for all water depths on 1380 thin top layers $k b \lesssim 0.1$.

As expected, for weaker currents, the same trends $13 \mathbf{1 3 8 2}$ are observed as mentioned already but the errors be- ${ }_{1383}$ come smaller for weaker currents.

[1] D. Bellafiore and G. Umgiesser. Hydrodynamic coastal 1385 processes in the North Adriatic investigated with a $3 \mathrm{~d} \quad \mathbf{1 3 8 6}$ finite element model. Ocean Dynamics, 60:255-273, 2010. 1387

[2] L. Cavaleri, B. Fox-Kemper, and M. Hemer. Wind waves 1388 in the coupled climate system. BAMS, pages 1651-1661, 1389 2012 . 46

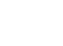

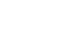
. 

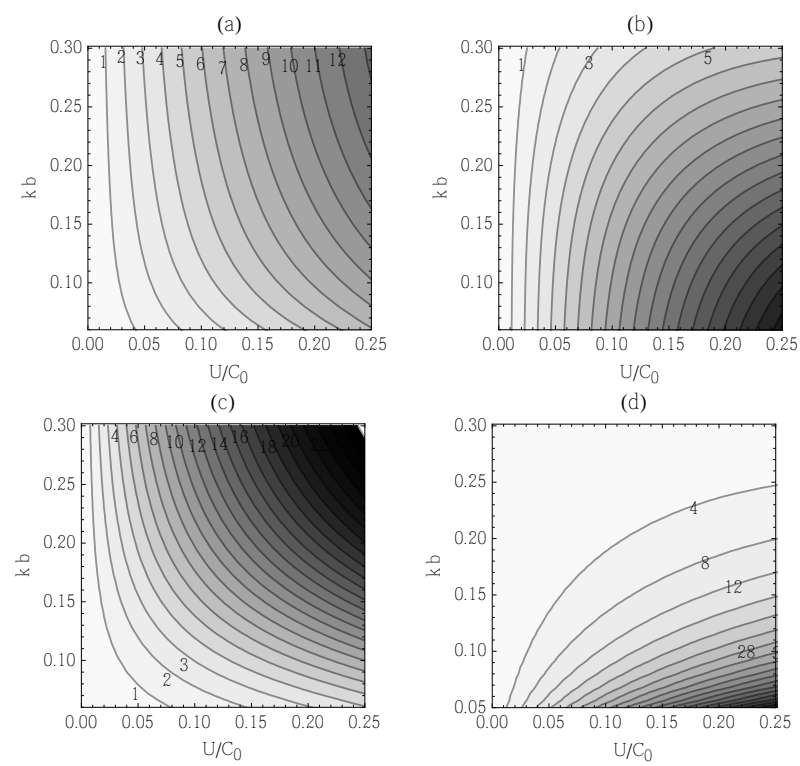

(e)

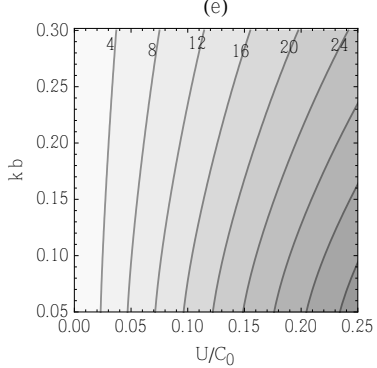

Figure F.8: Co-propagating waves with idealised two-layer vertical profile. Contours of (a) \%-errors of the approximated group velocity to the exact group velocity $C_{g} / C_{g v s}$, (b) the no-shear group velocity to the exact group velocity $C_{g_{n v s}} / C_{g v s},(\mathrm{c})$ the no-shear group velocity calculated with the depth-averaged velocity to the exact group velocity $\left(\left.C_{g_{n v s}}\right|_{U m}\right) / C_{g v s}$, (d) $I / I_{v s}$ and (e) $I_{n v s} / I_{v s}$, for $h=50 m$ $b / h=0.1$ and a variation of $k$ and $U$.

[3] F. P. Bretherton and C. J. R. Garrett. Wavetrains in inhomogeneous moving media. Proc. Roy. Soc. A, 302:5291969, 1968.

[4] A. G. Voronovich. Propagation of internal and surface gravity waves in the approximation of geometrical optics. Atmospheric and Oceanic Physics, 12:850-857, 1976.

[5] B. S. White. Wave action on currents with vorticity. J. of Fluid Mech., 386:329-344, 1999.

[6] O. Zikanov, D. N. Slinn, and M. R. Dhanak. Large-eddy simulations of the wind-induced turbulent Ekman layer. J. Fluid Mech., 495:343-368, 2003.

[7] J. A. Simmen and P. G. Saffman. Steady deep water waves on a linear shear current. Stud. Appl. Maths, 73:35-57, 1985.

[8] A. A. Abrashkin and D. A. Zen'kovich. Vortex steadystate waves on a shear flow. Fiz. Atmos. Okeana, 26(1):35-45, 1990.

[9] M. Oikawa, K. Chow, and D. J. Benney. Propagation of nonlinear wave packets in a shear flow with a free surface. Stud. Appl. Math., 76:69-92, 1987.

[10] V. E. Zakharov and V. I. Shrira. Formation of the angular spectrum of wind waves. Sov. Phys. JETP, 71:1091-1100, 1990.

[11] R. H. Stewart and J. W. Joy. HF radio measurements of surface currents. Deep-Sea Res., 21:1039-1049, 1974.

[12] R. A. Skop. Approximate dispersion relation for wavecurrent interactions. J. Waterway, Port, Coastal Eng., 113:1013-1027, 1987.

[13] V. I. Shrira. Surface waves on shear currents: solution of the boundary-value problem. J. Fluid Mech., 252:565-
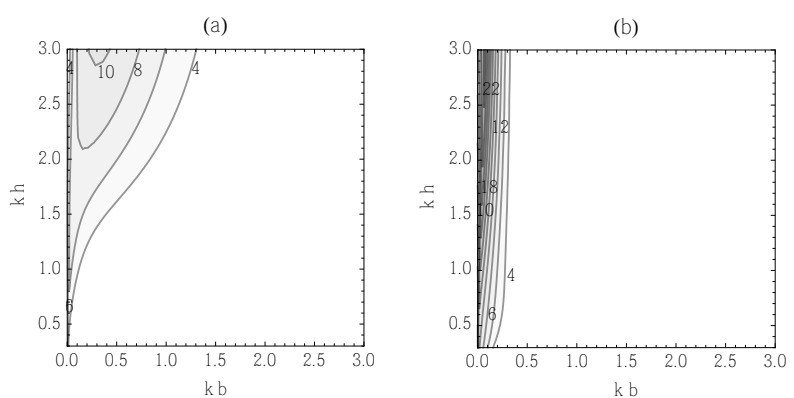

(c)
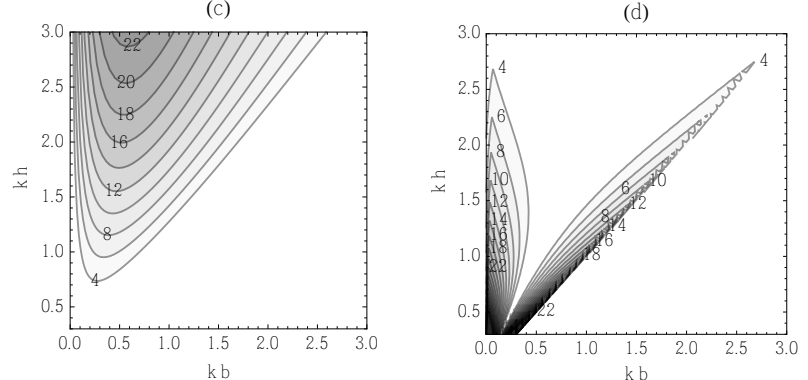

(e)

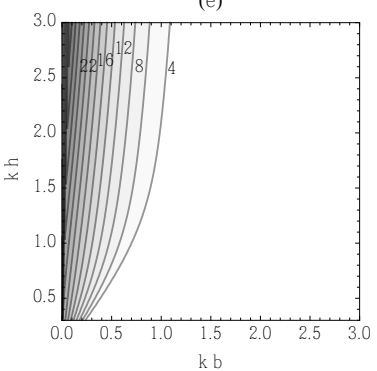

Figure F.9: Co-propagating waves with idealised two-layer vertical profile. Contours of (a) \%-errors of the approximated group velocity to the exact group velocity $C_{g} / C_{g v s}$, (b) the no-shear group velocity to the exact group velocity $C_{g_{n v s}} / C_{g v s}$, (c) the no-shear group velocity calculated with the depth-averaged velocity to the exact group velocity $\left(\left.C_{g_{n v s}}\right|_{U m}\right) / C_{g v s}$, (d) $I / I_{v s}$ and (e) $I_{n v s} / I_{v s}$, for $U(0)=$ $1.5 \mathrm{~ms}^{-1}, h=20 \mathrm{~m}$ and a variation of $b$ and $k$. The contours are at the same levels for each panel.

$584,1993$.

4] X. Zhang. Short surface waves on surface shear. J. Fluid $\mathbf{1 4 2 2}$ Mech., 541:345-370, 2005.

[15] D. H. Peregrine. Interaction of Water Waves and Cur- 1424 rents. Adv. Appl. Mech., 16:9-117, 1976. 6] D. H. Peregrine and I. G. Jonsson. Interaction of waves $\mathbf{1 4 2 6}$ and currents, pages Misc. Rep. 83-6. Coastal Eng. Res. 1427 Center. US Army Corps of Engs, Fort Belvoir, Virginia, 1428 1983.

[17] W. D. McKee. Waves on a shearing current: a uni- 140 formly valid asymptotic solution. Proc. Camb. Phil. Soc., $14 \mathbf{1 4 1}$ 75:295-301, 1974.

[18] W. D. McKee. The reflection of water waves by shear 1433 currents. PAGEOPH, 115:937, 1977. Mech., pages 401-416, 1978.

[20] S. A. Ellingson and I. Brevik. How linear surface waves ${ }_{1438}^{1437}$ are affected by a current with constant vorticity. Eur. J. 1439 Phys., 35:025005, 2014.

[21] J. Touboul, J Charland, V. Rey, and K. Belibassakis. Extended mild-slope equation for surface waves interacting $\mathbf{1 4 4 2}$ with a vertically sheared current. Coast. Eng., 116:77-88, 1443 2016.

[22] A. F. Teles Da Silva and Peregrine D. H. Steep, steady surface waves on water of finite depth with constant vorticity. J. Fluid Mech., 195:281-302, 1988.

[23] V. I. Shrira. Nonlinear waves at the surface of a liquid

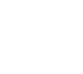


layer with a constant vorticity. Sov. Phys. Dokl., 31:107109, 1986.

[24] J. M. Vanden-Broeck. Steep solitary waves in water of finite depth with constant vorticity. J. Fluid Mech., 274:339-348, 1994.

[25] D. H. Peregrine and G. P. Thomas. Finite-Amplitude Deep-Water Waves on Currents. Proc. R. Soc. Lond., A. 292:371-390, 1979.

[26] V. P. Ruban. Explicit equations for two-dimensional water waves with constant vorticity. Phys. Rev. E, 77:037302, 2008.

[27] R. S. Johnson. On the modulation of water waves on shear flows. Proc. R. Soc. Lond., A. 347:537-546, 1976.

[28] A. I. Baumstein. Modulation of Gravity Waves with Shear in Water. Stud. Appl. Math., 100:365-390, 1998.

[29] R. Thomas, C. Kharif, and M. Manna. A nonlinear Schrödinger equation for water waves on finite depth with constant vorticity. Phys. Fluids, 24:127102, 2012.

[30] AG Voronovich and SA Rybak. Explosive instability of stratified flows. DOKLADY AKADEMII NAUK SSSR, 239(6):1457-1460, 1978.

[31] AG Voronovich, ED Lobanov, and SA Rybak. On the stability of gravitational-capillary waves in the presence of a vertically nonuniform current. Izv. Atmos. Ocean Phys, 16:220-222, 1980.

[32] Vladimir Kudryavtsev, Victor Shrira, Vladimir Dulov, and Vladimir Malinovsky. On the vertical structure of wind-driven sea currents. Journal of Physical Oceanography, 38(10):2121-2144, 2008.

[33] Alexander Babanin. Breaking and dissipation of ocean surface waves. Cambridge University Press, 2011.

[34] C. Swan and R. L. Cummins, I. P.and James. An experimental study of two-dimensional surface water waves propagating on depth-varying currents. Part 1. Regular waves. J. Fluid Mech., 428:273-304, 2001.

[35] C. M. Bender and S. A. Orszag. Advanced Mathematical Methods for Scientists and Engineers I. Asymptotic Methods and Perturbation Theory. McGraw Hill, 1978.

[36] P. J. Schmid and D. S. Henningson. Stability and Transition in Shear Flows. Springer-Verlag, 2001.

[37] J. T. Kirby and T.-M. Chen. Surface waves on vertically sheared flows: approximate dispersion relations. J. Geophys. Res., 94:1013-1027, 1989.

[38] J. Wu and I. K. Tsanis. Numerical study of wind-induced water currents. J. Hydraul. Eng., 121:388-395, 1995.

[39] C. C. Mei, M. Stiassnie, and D. K. P. Yue. Theory and Applications of ocean surface waves. Part 1: Linear aspects. World Scientific, 2005.

[40] L. M. Brekhovskikh and O. A. Godin. Acoustics of Layered Media II. Springer-Verlag, 1992.

[41] Victor I Shrira and Igor A Sazonov. Quasi-modes in boundary-layer-type flows. part 1 . inviscid twodimensional spatially harmonic perturbations. Journal of Fluid Mechanics, 446:133-171, 2001

[42] Jennifer Waters, Lucy R. Wyatt, Judith Wolf, and Adrian Hines. Data assimilation of partitioned $\{\mathrm{HF}\}$ radar wave data into wavewatch $\{\mathrm{III}\}$. Ocean Modelling, 72:17 - 31, 2013.

[43] VP Krasitskii. On reduced hamiltonian equations in the nonlinear theory of water surface waves. J. Fluid Mech, 272:1-20, 1994.

[44] A. H. Nayfeh. Introduction to Perturbation Techniques. Wiley-Interscience, 1993.

[45] J. S. Turner. Buoyancy effects in fluids. Cambridge University Press, 1973. 